\title{
Hans Lycke*
}

\section{INCONSISTENCY-ADAPTIVE MODAL LOGICS On How to Cope with Modal Inconsistency}

\begin{abstract}
In this paper, I will characterize a new class of inconsistencyadaptive logics, namely inconsistency-adaptive modal logics. These logics cope with inconsistencies in a modal context. More specifically, when faced with inconsistencies, inconsistency-adaptive modal logics avoid explosion, but still allow the derivation of sufficient consequences to adequately explicate the part of human reasoning they are intended for.
\end{abstract}

Keywords: modal logics, paraconsistency, inconsistency-adaptive logics, inconsistency-adaptive modal logics.

\section{Introduction}

Paraconsistent modal logics (pML, see e.g. [12, 14, 22]) are obtained by adding the modal operators $\square$ (necessity) and $\diamond$ (possibility) to a paraconsistent logic. As usual, the modal operators are interpreted as deontic, alethic, doxastic, epistemic, ... operators, depending on their specific characteristics. Moreover, as $\mathbf{p M L}$ are based on paraconsistent logics, they do not validate Ex Falso Quodlibet (EFQ: $A, \sim A / B$ ). Hence, pML combine the expressive power of modal logics with the non-explosive character of paraconsistent logics. As such, they seem well-suited to

*Post-doctoral Fellow of the Special Research Fund of Ghent University. I am indebted to two anonymous referees for helpful comments on a previous version of this paper. Moreover, I would also like to thank Koji Tanaka, Dagmar Provijn, Bert Leuridan and Steffen Ducheyne for helpful suggestions. 
explicate normative reasoning, reasoning about beliefs, reasoning about knowledge, .... In all of these, people frequently come to inconsistent conclusions, without leaping into triviality (e.g. holding inconsistent beliefs isn't equal to believing everything).

Despite the absence of EFQ, paraconsistent modal logics do not capture the intended part of human reasoning in a realistic way. For, they reach paraconsistency by invalidating some of the classical rules of inference, for example modus ponens $(A, A \supset B / B)$, disjunctive syllogism $(A \vee B, \sim A / B)$, modus tollens $(A \supset B, \sim B / \sim A)$, the de Morgan laws, .... These inference rules are central to human reasoning. They are frequently used and they hardly ever lead to trivial consequences. Hence, as $\mathbf{p M L}$ do not validate these inference rules, they do not have sufficient deductive strength to explicate human reasoning in an adequate way.

The above problem is not specific to $\mathbf{p M L}$, but applies to paraconsistent logics in general. Notable exceptions are inconsistency-adaptive logics (iAL, see e.g. [1, 2, 9]). The latter do not invalidate the above inference rules in general, but only invalidate their problematic applications (the applications that, if allowed, would lead to trivial consequences). Hence, when applied to consistent premise sets, iAL validate all applications, for none of them is problematic in this case. This means that $\mathbf{i A L}$ only invalidate those applications of the classical inference rules that are problematic for the specific premise set they are applied to. As a consequence, $\mathbf{i A L}$ are said to oscillate between a paraconsistent lower limit logic (allowing no applications of some classical inference rules) and an explosive upper limit logic (allowing all applications of the classical inference rules). In that way, they combine paraconsistency with sufficient deductive power to capture human reasoning in a more realistic way.

Up to now, only iAL with a non-modal lower limit logic have been developed. If modal logics were used within the scope of $\mathbf{i A L}$, this was solely to characterize (non-modal) paraconsistent inference relations (or better, inconsistency handling mechanisms) under a (modal) translation, and not to extend the inconsistency-adaptive approach to modal logics as such (see e.g. $[4,6,8,11,17,18,19,23])$. In this paper, I will show that the inconsistency-adaptive framework can be extended to modal logics as well. Basically, I will do so by presenting the inconsistency-adaptive modal logics $\mathbf{i} \mathbf{A T} \overline{\mathbf{u}} \mathbf{N} \mathbf{s}_{\text {syn }}^{\mathbf{r}}$ and $\mathbf{i} \mathbf{A} \mathbf{T} \overline{\mathbf{u}} \mathbf{N} \mathbf{s}_{\mathbf{s e m}}^{\mathbf{r}}$. Both oscillate between the paraconsistent modal logic $\mathbf{T} \overline{\mathbf{u}} \mathbf{N s}$ (the lower limit logic) and the explosive modal logic $\mathbf{T}$ (the upper limit logic). Moreover, as other inconsistency- 
adaptive modal logics are characterized equivalently, the result is a new class of iAL: the class of inconsistency-adaptive modal logics (iAML).

In accordance to their non-modal counterparts, iAML only invalidate those applications of some inference rules of standard modal logics that are problematic in view of the particular premise set they are applied to. Hence, in comparison with other paraconsistent modal logics, this new class of $\mathbf{i A L}$ enables us to explicate more realistically that part of human reasoning that makes use of modal operators (i.e. reasoning about beliefs, knowledge, laws, ....).

Overview. In Section 2, I will give a short, intuitive characterization of (non-modal) iAL. In Section 3, I will present the inconsistency-adaptive modal framework by characterizing the inconsistency-adaptive modal logics $\mathbf{i} A \mathbf{T} \overline{\mathbf{u}} \mathbf{N} \mathbf{s}_{\mathbf{s y n}}^{\mathbf{r}}$ and $\mathbf{i} \mathbf{A T} \overline{\mathbf{u}} \mathbf{N s}_{\text {sem }}^{\mathbf{r}}$ (sections 3.1-3.3). Moreover, I will also point out a peculiar feature of those inconsistency-adaptive modal logics that are characterized semantically by means of a non-reflexive accessibility relation (Section 3.4). Finally, in Section 4, I will present a meaningful application of the inconsistency-adaptive modal framework.

\section{Inconsistency-Adaptive Logics}

As adaptive logics (AL) include, but are not restricted to inconsistencyadaptive logics (a common misunderstanding about adaptive logics), I will first give a characterization of $\mathbf{A L}$ in general. Next, I will turn to inconsistency-adaptive logics in particular. The former will be done by presenting the standard format of $\mathbf{A L}$ (see also $[3,5]$ ), the latter by specifying how inconsistency-adaptive logics fit the standard format of AL (see also $[1,2,9])$.

The Standard Format. All (standard) adaptive logics are fully characterized by three elements: a lower limit logic (LLL), a set of abnormalities $\Omega$ (a set of formulas characterized by a logical form F), and an adaptive strategy.

The LLL is the stable part of an adaptive logic, which comes down to the fact that all $\mathbf{L L L}$-consequences of a premise set are also $\mathbf{A L}$ consequences of that premise set. Proof theoretically, this means that in AL-proofs, all LLL-inference rules may be applied unrestrictedly. 
However, an adaptive logic typically enables to derive more consequences from a premise set than its LLL. The supplementary ALconsequences are obtained by interpreting a premise set as normally as possible. This is done by interpreting as false as many elements of $\Omega$ (abnormalities) as possible. More specifically, this comes down to the fact that some formulas are conditionally derivable from a premise set: in case $\Gamma \vdash_{\mathbf{L L L}} A \vee \operatorname{Dab}(\Delta)$, with $\operatorname{Dab}(\Delta)$ a $D a b$-formula (a finite disjunction of abnormalities), then $\Gamma \vdash_{\text {AL }} A$, unless there are reasons to consider some elements of $\Delta$ as true (in which case $A$ cannot safely be considered to be derivable from $\Gamma$ ). Proof theoretically, this means that some conditional inference rules are added to the LLL-proof theory. These may be applied unless or until there are reasons to consider their consequences as problematic.

Which of the conditional consequences of a premise set $\Gamma$ are also final consequences of $\Gamma$ (i.e. actual AL-consequences of $\Gamma$ ), depends on the $D a b$-consequences of $\Gamma$. The latter are the $D a b$-formulas that are LLL-derivable from $\Gamma$. Obviously, not all abnormalities occurring in a $D a b$-consequence can be considered as false. Otherwise, the $D a b$ consequence itself cannot possibly be considered as true. Hence, some of the conditional consequences will have to be rejected (because they were derived by mistakenly presupposing some of the abnormalities in a Dab-consequence to be false). Which of them will eventually be rejected, is determined by the adaptive strategy. Moreover, as different strategies might reject different conditional consequences, the adaptive strategy can be regarded as the guideline of how to cope with the Dab-consequences of a premise set.

Notice that in case no Dab-formulas are LLL-derivable from a premise set, it is safe to consider all abnormalities as false. As a consequence, the adaptive logic will then yield the same consequence set as the logic that interprets all abnormalities as false unconditionally (or equivalently, as the logic that fully validates the inference rules whose application the adaptive logic only allows conditionally). This logic is called the upper limit logic (ULL) of an adaptive logic. In general, the ULL is related to the LLL as set out by the Derivability Adjustment Theorem (DAT):

Theorem 1 (DAT). $\Gamma \vdash_{\mathbf{U L L}} A$ iff there is some finite $\Delta \subset \Gamma$ such that $\Gamma \vdash_{\text {LLL }} A \vee \operatorname{Dab}(\Delta)$. 
How close the AL-consequence set will come to the ULL-consequence set, depends on the premise set the adaptive logic is applied to. The less $D a b$-consequences derivable from the premise set, the closer the $\mathbf{A L}$ consequence set will come to the ULL-consequence set. This is why adaptive logicians say that $\mathbf{A L}$ adapt themselves to their premise sets.

The Inconsistency-Adaptive Framework. As mentioned in the previous section, inconsistency-adaptive logics (iAL) are based on a paraconsistent LLL. Hence, all consequences obtained from a premise set by this paraconsistent logic are also $\mathbf{i A L}$-consequences of that premise set. More iAL-consequences are obtained by interpreting a premise set as consistently as possible. In fact, this means that iAL presuppose inconsistencies to be false unless there are reasons for not doing so, reasons provided by those disjunctions of inconsistencies that are LLL-derivable from the premise set. Obviously, this implies that the set of abnormalities $\Omega$ of an inconsistency-adaptive logic is the set of all inconsistencies (possibly limited to those of a restricted form). As a consequence, the ULL of an inconsistency-adaptive logic is a logic that presupposes inconsistencies to be false unconditionally. In other words, the ULL of an inconsistencyadaptive logic is an explosive logic (in most cases, it is even classical $\operatorname{logic})$.

\section{Inconsistency-Adaptive Modal Logics}

There are two ways to extend the inconsistency-adaptive framework to modal logics. I have called these respectively the syntactic and the semantic approach to modal inconsistency. To characterize both approaches, I will present the inconsistency-adaptive modal logics $\mathbf{i A T} \overline{\mathbf{u}} \mathbf{N} s_{\text {syn }}^{\mathbf{r}}$ and $\mathbf{i A T} \overline{\mathbf{u}} \mathbf{N} \mathbf{s}_{\text {sem }}^{\mathbf{r}}$. The former is obtained by means of the syntactic approach, the latter by means of the semantic approach.

Overview. First, I will characterize the paraconsistent modal logic $\mathbf{T} \overline{\mathbf{u}} \mathbf{N}$ s that will serve as the $\mathbf{L L L}$ of both $\mathbf{i A T} \overline{\mathbf{u}} \mathbf{N} \mathbf{s}_{\mathbf{s y n}}^{\mathbf{r}}$ and $\mathbf{i A T} \overline{\mathbf{u}} \mathbf{N} \mathbf{s}_{\text {sem }}^{\mathbf{r}}$ (in Section 3.1). Next, I will give an intuitive introduction to both the syntactic as well as the semantic approach to modal inconsistency (in Section 3.2). After that, I will present both the semantics and the proof theory of the inconsistency-adaptive modal logics $\mathbf{i A T} \overline{\mathbf{u}} \mathbf{N} \mathbf{s}_{\mathbf{s y n}}^{\mathbf{r}}$ and $\mathbf{i A T} \overline{\mathbf{u}} \mathbf{N s}_{\text {sem }}^{\mathrm{r}}$, obtained by applying respectively the syntactic and the 
semantic approach to modal inconsistency (in Section 3.3). Finally, I will point out a peculiar feature of the inconsistency-adaptive modal logics whose LLL is characterized by means of a non-reflexive accessibility relation (in Section 3.4).

\subsection{The Paraconsistent Modal Logic TūNs}

The paraconsistent modal logic $\mathbf{T} \overline{\mathbf{u}} \mathbf{N s}$ is a modal extension of the paraconsistent logic $\mathbf{C L u} \mathbf{u}$ s (see [7],[16, ch. 4]). ${ }^{1} \quad$ Moreover, as the logic $\mathbf{T} \overline{\mathbf{u}} \mathbf{N s}$ is semantically characterized by means of a reflexive accessibility relation (see below), it is related to $\mathbf{C L} \overline{\mathbf{u}} \mathbf{N s}$ in the same way as the (explosive) modal logic $\mathbf{T}$ is related to classical logic. Hence, the logic $\mathbf{T} \overline{\mathbf{u}} \mathbf{N} \mathbf{s}$ can be considered as the paraconsistent counterpart of the logic $\mathbf{T}$.

Language Schema. Let $\mathcal{L}^{\mathcal{M}}$ be the standard propositional modal language with $\mathcal{S}, \mathcal{S}^{\sim}$ and $\mathcal{W}^{\mathcal{M}}$ the sets of sentential letters, negations of sentential letters, and well-formed formulas respectively. Negation, conjunction and necessity are taken as primitive, while the other logical symbols are defined in the usual way.

Semantics. A $\mathbf{T} \overline{\mathbf{u}} \mathbf{N s}$-model $M$ for the language $\mathcal{L}^{\mathcal{M}}$ is a 4 -tuple $\left\langle w_{0}\right.$, $W, R, v\rangle$, with $W$ a set of worlds, $w_{0}$ the actual world, $R$ a reflexive accessibility relation on $W$, and $v: \mathcal{S} \cup \mathcal{S}^{\sim} \times W \longrightarrow\{0,1\}$ an assignment function.

The valuation function $v_{M}: \mathcal{W}^{\mathcal{M}} \times W \longrightarrow\{0,1\}$, determined by the model $M$, is defined by means of the following semantic postulates:

SP1 For $A \in \mathcal{S}: v_{M}(A, w)=1$ iff $v(A, w)=1$.

SP2 For $A \in \mathcal{S}: v_{M}(\sim A, w)=1$ iff $v_{M}(A, w)=0$ or $v(\sim A, w)=1$.

SP3 $v_{M}(\sim \sim A, w)=1$ iff $v_{M}(A, w)=1$.

SP4 $v_{M}(A \wedge B, w)=1$ iff $v_{M}(A, w)=1$ and $v_{M}(B, w)=1$.

SP5 $v_{M}(\sim(A \wedge B), w)=1$ iff $v_{M}(\sim A, w)=1$ or $v_{M}(\sim B, w)=1$.

SP6 $v_{M}(\square A, w)=1$ iff for all $w^{\prime} \in W$, if $R w w^{\prime}$ then $v_{M}\left(A, w^{\prime}\right)=1$.

SP7 $v_{M}(\sim \square A, w)=1$ iff for some $w^{\prime} \in W, R w w^{\prime}$ and $v_{M}\left(\sim A, w^{\prime}\right)=1$.

A model $M$ verifies a formula $A \in \mathcal{W}^{\mathcal{M}}$ iff $v_{M}\left(A, w_{0}\right)=1$. Also, a model $M$ is a model of a premise set $\Gamma$ iff, for all $B \in \Gamma, v_{M}\left(B, w_{0}\right)=1$.

\footnotetext{
${ }^{1}$ In fact, $\mathbf{C L \overline { u } N s}$ is equivalent to Priest's well-known LP (see [20],[21, ch. 7-8]), but is characterized quite differently.
} 
Finally, semantic consequence for the logic $\mathbf{T} \overline{\mathbf{u}} \mathbf{N} \mathbf{s}$ is defined as truth preservation at the actual world $w_{0}$ :

Definition 1. $\Gamma \vDash_{\mathbf{T u}}$ us $A$ ( $A$ is a $\mathbf{T} \overline{\mathbf{u}} \mathbf{N}$ s-consequence of $\Gamma$ ) iff $A$ is verified by all $\mathbf{T} \overline{\mathbf{u}} \mathbf{N s}$-models of $\Gamma$.

Proof Theory. I will present a Fitch-style natural deduction proof theory for the logic $\mathbf{T} \overline{\mathbf{u}} \mathbf{N s}$. Before spelling out the proof theoretic rules, two important remarks have to be made. ${ }^{2}$

First of all, the proof theory allows for two kinds of subproofs: classical subproofs and modal subproofs. The former are the standard kind of subproofs, well-known from classical logic. The latter are specific for (paraconsistent) modal logics. They show what would follow from the hypothesis if it were true in some arbitrary world (see the structural rules HYP and HYP ${ }^{\square}$ below).

Secondly, TūNs-proofs do not only make use of well-formed formulas (wffs). They also make use of pseudo-formulas:

Definition 2. If $A, B \in \mathcal{W}^{\mathcal{M}}$ then $S(A, B)$ and $S^{\square}(A, B)$ are pseudoformulas of the modal language $\mathcal{L}^{\mathcal{M}}$.

The pseudo-formulas $S(A, B)$ and $S^{\square}(A, B)$ express "the formula $B$ is derivable from the formula $A$ in this world" and "the formula $B$ is derivable from the formula $A$ in any world" respectively. Hence, they are used in the proof theory to represent the conclusions that can be drawn from classical and modal subproofs respectively (see the inference rules CSP and CSP $\square$ below).

Proof Theoretic Rules. First, consider the structural rules of the TūNsproof theory.

PREM Premises may be written down at any place in the main proof. HYP At any place in the proof, one may start a new classical subproof. This is done by introducing a new hypothesis, together with a new vertical line on its left.

$$
\begin{array}{l|ll}
\mathrm{i} & A & -; \mathrm{HYP} \\
\ldots & \ldots & \ldots
\end{array}
$$

\footnotetext{
${ }^{2}$ The remarks are kept quite intuitive, which will do for the purposes of this paper. For a full-fledged characterization of this proof theory, see [15].
} 
HYP $\square$ At any place in the proof, one may start a new modal subproof. This is done by introducing a new hypothesis, together with a new vertical line on its left. Moreover, to distinguish a modal subproof from a classical one, a $\square$-symbol is placed next to its vertical line.

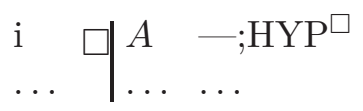

REP In the main proof and in both classical and modal subproofs, formulas (and pseudo-formulas) may be repeated.

REIT Reiteration is restricted to classical subproofs. Hence, formulas (and pseudo-formulas) may be reiterated in unclosed classical subproofs, but not in modal subproofs.

Next, consider the $\mathbf{T} \overline{\mathbf{u}} \mathbf{N s}$-inference rules. Those presented by means of a double vertical line ( ||) allow for derivation in both directions, while the others only allow for left-right derivation.

CSP If the formula $B$ is the formula on the last line of a classical subproof that started with the hypothesis $A$, one may conclude the pseudo-formula $S(A, B)$.

$\mathrm{CSP}^{\square}$ If the formula $B$ is the formula on the last line of a modal subproof that started with the hypothesis $A$, one may conclude the pseudo-formula $S^{\square}(A, B)$.

\begin{tabular}{|c|c|c|c|}
\hline $\mathrm{CON}$ & $A, B \mid A \wedge B$ & $\mathrm{CON}^{\diamond}$ & $\square A \wedge \diamond B \mid \diamond(A \wedge B)$ \\
\hline SIM & $A \wedge B|A ; A \wedge B| B$ & $\mathrm{CON}^{\square}$ & $\square A \wedge \square B$ \\
\hline $\mathrm{ADD}$ & $A|A \vee B ; B| A \vee B$ & $\mathrm{DIS}^{\square}$ & $\square(A \vee B)$ \\
\hline DIL & $A \vee B, S(A, C), S(B, C) \mid C$ & $\mathrm{DIS}^{\diamond}$ & $\diamond(A \vee B) \mid \diamond A \vee \diamond B$ \\
\hline IMP & $A \supset B \| \sim A \vee B$ & $\mathrm{MP}^{\square}$ & $\square A, S^{\square}(A, B) \mid \square B$ \\
\hline DN & $\sim \sim A \| A$ & $\mathrm{MP}^{\diamond}$ & $\diamond A, S^{\square}(A, B) \mid \diamond B$ \\
\hline $\mathrm{NC}$ & $\sim(A \wedge B) \| \sim A \vee \sim B$ & $\mathrm{NEG}^{\square}$ & $\sim \square A \| \diamond \sim A$ \\
\hline ND & $\sim(A \vee B) \| \sim A \wedge \sim B$ & $\mathrm{NEG}^{\diamond}$ & $\sim \diamond A \| \square \sim A$ \\
\hline NI & $\sim(A \supset B) \| \sim A \wedge B$ & NEC & $A \in \mathcal{S}: A \vee \sim A \mid \square(A \vee \sim A)$ \\
\hline & $A \in \mathcal{S}: \emptyset \mid A \vee \sim A$ & & \\
\hline
\end{tabular}

It is easily verified that the inference rule DIL' below is a derived rule of inference of the logic $\mathbf{T} \overline{\mathbf{u}} \mathbf{N s}$.

$\mathrm{DIL}^{\prime} \quad A \vee B, S(A, C)|C \vee B ; A \vee B, S(B, C)| A \vee C$ 
Proof. Consider the generic $\mathbf{T} \overline{\mathbf{u}} \mathbf{N s}$-proof below. It not only proves that DIL' is a derivable inference rule of $\mathbf{T} \overline{\mathbf{u}} \mathbf{N} \mathbf{s}$, it also illustrates the proof theory of $\mathbf{T} \overline{\mathbf{u}} \mathbf{N s}$ quite nicely.

$\begin{array}{lll}1 & A \vee B & \mathrm{PREM} \\ \ldots & \ldots & \ldots \\ \mathrm{i} & S(A, C) & \ldots \\ \mathrm{i}+1 & B & \mathrm{HYP} \\ \mathrm{i}+2 & C \vee B & \mathrm{i}+1 ; \mathrm{ADD} \\ \mathrm{i}+3 & S(B, C \vee B) & \mathrm{i}+1, \mathrm{i}+2 ; \mathrm{CSP} \\ \mathrm{i}+4 & A & \mathrm{HYP} \\ \mathrm{i}+5 & A \vee A & \mathrm{i}+4 ; \mathrm{ADD} \\ \mathrm{i}+6 & S(A, C) & \mathrm{i} ; \mathrm{REIT} \\ \mathrm{i}+7 & S(A, C) & \mathrm{i}+6 ; \mathrm{REP} \\ \mathrm{i}+8 & C & \mathrm{i}+5, \mathrm{i}+6, \mathrm{i}+7 ; \mathrm{DIL} \\ \mathrm{i}+9 & C \vee B & \mathrm{i}+8 ; \mathrm{ADD} \\ \mathrm{i}+10 & S(A, C \vee B) & \mathrm{i}+4, \mathrm{i}+9 ; \mathrm{CSP} \\ \mathrm{i}+11 & C \vee B & 1, \mathrm{i}+3, \mathrm{i}+10 ; \mathrm{DIL} \quad \dashv\end{array}$

Derivability. A T TūNs-proof is defined as a finite sequence of wffs (and pseudo-wffs), each of which is either a premise or follows from wffs (and pseudo-wffs) earlier in the list by means of a rule of inference. Moreover, in order for such a sequence to be a proof, all its subproofs have to be closed.

Finally, T $\mathbf{u} \mathbf{N s}$-derivability is defined as follows:

Definition 3. $\Gamma \vdash_{\mathbf{T}} \mathbf{\mathbf { u } N \mathbf { s }} A$ ( $A$ is $\mathbf{T} \overline{\mathbf{u}} \mathbf{N s}$-derivable from $\Gamma$ ) iff there is a proof of the formula $A$ from $B_{1}, \ldots, B_{n} \in \Gamma$ so that $A$ has been derived on a line $i$ of the main proof.

Soundness and Completeness. Soundness and completeness proofs for the logic $\mathbf{T} \overline{\mathbf{u}} \mathbf{N s}$ can be found in [15]. Hence, they will not be repeated here.

TheOrem 2. $\Gamma \vDash_{\text {TūNs }} A$ iff $\Gamma \vdash_{\text {TūNs }} A$.

Explosion Reconsidered. In T $\overline{\mathbf{u}} \mathbf{N s}$-models, the assignment function assigns a truth value to both sentential letters and their negations. Some valuation functions make use of this feature to assign truth to both a 
sentential letter and its negation (by SP2). The result is a paraconsistent negation. To obtain an explosive negation, SP2 has to be replaced by SP2c below. The latter characterizes the negation classically, whence the resulting logic is explosive.

SP2c $\quad$ For $A \in \mathcal{S}: v_{M}(\sim A, w)=1$ iff $v_{M}(A, w)=0$.

More specifically, the resulting logic is the normal modal logic $\mathbf{T}$ of Feys (von Wright's $\mathbf{M}$ ). Moreover, the proof theory of the logic $\mathbf{T}$ is obtained by adding the following inference rules to the proof theory of the logic TūNs.

DS For $A \in \mathcal{S}: B \vee(A \wedge \sim A) \mid B$

INC $\quad$ For $A \in \mathcal{S}: \diamond(A \wedge \sim A) \mid A \wedge \sim A$

The soundness and completeness proofs of the logic $\mathbf{T}$ are similar to those of the logic $\mathbf{T} \overline{\mathbf{u}} \mathbf{N}$ s and can also be found in [15].

To conclude, it is necessary to point out the specific relation between the paraconsistent logic $\mathbf{T} \overline{\mathbf{u}} \mathbf{N s}$ and the explosive logic $\mathbf{T}$. First, consider the set $\mathcal{I} n c$, the set of all possible inconsistencies (more specifically, all possible inconsistencies that consist of a sentential letter and its negation).

Definition 4. Inc $=\{A \wedge \sim A \mid A \in \mathcal{S}\} \cup\left\{\diamond \ldots \diamond(A \wedge \sim A) \in \mathcal{W}^{\mathcal{M}} \mid\right.$ $A \in \mathcal{S}\}$.

Next, consider Theorem 3. It states that the formula $A$ is derivable from a premise set by means of the logic $\mathbf{T}$ iff a disjunction of $A$ and some members of $\mathcal{I} n c$ is derivable from that premise set by means of the $\operatorname{logic} \mathbf{T} \overline{\mathbf{u}} \mathbf{N s}$.

Theorem 3. For a finite $\Delta \subset \mathcal{I} n c, \Gamma \vdash_{\mathbf{T}} A$ iff $\Gamma \vdash_{\mathbf{T} u \bar{N} \mathbf{s}} A \vee \bigvee(\Delta) .^{3}$

Proof. Right-Left. Obvious in view of the inference rules INC and DS.

Left-Right. Any $\mathbf{T}$-proof of a formula $A$ can be converted into a T $\overline{\mathbf{u}}$ Ns-proof of a formula $A \vee \bigvee(\Delta)$ by making use of the inference rules $\mathrm{DIS}^{\square}, \mathrm{DIS}^{\diamond}, \mathrm{MP}^{\square}, \mathrm{MP}^{\diamond}$ and the derived rule of inference DIL'. Details are left to the reader.

\footnotetext{
${ }^{3}$ Obviously, $\bigvee(\Delta)$ represents the disjunction of all members of $\Delta$.
} 


\subsection{How to Cope with Modal Inconsistency?}

In order to specify the inconsistency-adaptive modal framework, the inconsistency-adaptive modal logics iAT $\overline{\mathbf{u}} \mathbf{N} \mathbf{s}_{\mathbf{s y n}}^{\mathbf{r}}$ and $\mathbf{i A T} \overline{\mathbf{u}} \mathbf{N} \mathbf{s}_{\mathbf{s e m}}^{\mathbf{r}}$ will be characterized. Both oscillate between the paraconsistent lower limit logic $\mathbf{T} \overline{\mathbf{u}} \mathbf{N s}$ and the explosive upper limit logic $\mathbf{T}$. Hence, the lower limit logic of both logics is the same. Moreover, both logics are also based on the same adaptive strategy, namely the reliability strategy. ${ }^{4}$ As a consequence, the difference between both logics solely has to do with the way the abnormalities are defined. For $\mathbf{i} \mathbf{A T} \overline{\mathbf{u}} \mathbf{N s}_{\mathbf{s y n}}^{\mathbf{r}}$, they are defined by syntactic means (the syntactic approach to modal inconsistency), while for $\mathbf{i A T} \overline{\mathbf{u}} \mathbf{N s} \mathbf{s e m}_{\mathbf{s e m}}^{\mathbf{r}}$, they are defined by semantic means (the semantic approach to modal inconsistency).

Before focussing on each approach separately, it is necessary to stress that, as the resulting logics fall within the inconsistency-adaptive framework, the general aim of both approaches is to interpret a premise set as consistently as possible. This means that the abnormalities defined by the approaches are inconsistencies. More specifically, they are modal inconsistencies. Hence, because both approaches are based on different sets of abnormalities, they not only represent two distinct ways to cope with modal inconsistency, they also represent two distinct ways to define what it is for a modal premise set to be inconsistent.

The Syntactic Approach. Because of the equivalence between Theorem 3 (see Section 3.1) and the Derivability Adjustment Theorem of adaptive logics (see Section 2), it seems quite natural to define the set of abnormalities of inconsistency-adaptive modal logics as identical to the set Inc (see Definition 4, Section 3.1). This is exactly what is done by the syntactic approach.

Definition 5. $\Omega_{\text {syn }}=\{A \wedge \sim A \mid A \in \mathcal{S}\} \cup\left\{\diamond \ldots \diamond(A \wedge \sim A) \in \mathcal{W}^{\mathcal{M}} \mid\right.$ $A \in \mathcal{S}\}$.

Remember that the elements of $\Omega_{\text {syn }}$ (henceforth, abnormalities ${ }_{s y n}$ ) are taken to be modal inconsistencies. Hence, the possibility opera-

\footnotetext{
${ }^{4}$ Because of space limitations, only the reliability strategy is considered in this paper. However, inconsistency-adaptive modal logics can also be based on other strategies, such as for example minimal abnormality, normal selections, counting, .... For more information on those strategies, consult (among others) [5, 10, 23].
} 
tors that might occur in them cannot be interpreted as operators that point to an inconsistency (or to an inconsistent world). They have to be considered an integral part of the inconsistency itself. Obviously, this is quite counterintuitive, for it is a longstanding tradition to identify inconsistencies with formulas of the form $B \wedge \sim B$. Of course, the counterintuitive result is due to the fact that the set of abnormalities is determined purely on syntactic grounds. Nevertheless, this is not the only reason. It is also due to the fact that (explosive) modal logics yield the trivial consequence set for premise sets from which formulas of the form $\diamond \ldots \diamond(B \wedge \sim B)$ are derivable. This is at least as counterintuitive, for why should triviality be the result of inconsistencies that are merely possible, and not actual? Hence, it might be better to call the elements of $\Omega_{\text {syn }}$ triviality-generating formulas instead of inconsistencies. If this is done, the syntactic approach to modal inconsistency makes perfect sense: it interprets as many triviality-generating formulas as possible as false. Hence, the syntactic approach to modal inconsistency (I will keep the name!) interprets a premise set as free of triviality-generating formulas as possible. ${ }^{5}$

The Semantic Approach. Instead of focussing on triviality-generating formulas (as does the syntactic approach), the semantic approach to modal inconsistency focusses on standard inconsistencies, whence on formulas of the form $B \wedge \sim B$. Hence, the set of abnormalities of inconsistency-adaptive modal logics obtained by means of the semantic approach is defined as follows:

Definition 6. $\Omega_{\text {sem }}=\{A \wedge \sim A \mid A \in \mathcal{S}\}$.

However, because of the modal (hence, possible world) context, more needs to be said. For, the semantic approach does not focuss on inconsistencies as such. It focusses on reachable inconsistencies, which are inconsistencies (elements of $\Omega_{\text {sem }}$ ) that are true in a reachable world. A reachable world is a world that is somehow connected with the actual world $w_{0}$. More specifically, a world $w_{n}$ is considered as reachable, when-

\footnotetext{
${ }^{5} \mathrm{I}$ am aware of the fact that the term "triviality-generating formula" might not be the best one around to refer to elements of $\Omega_{\text {syn }}$. For, in paraconsistent modal logics, they do not generate triviality at all (on the contrary, an inconsistency remains an inconsistency, whether it generates triviality or not). However, it is the best term I could come up with. Hence, it has to do, at least for this paper.
} 
ever it is possible to go from $w_{0}$ to $w_{n}$ in a finite number of steps, by jumping from one accessible world to another. Hence, the semantic approach to modal inconsistency interprets a premise set as consistently as possible by interpreting the set of all reachable worlds as consistently as possible. In other words, the approach interprets as many reachable inconsistencies as possible as false.

Obviously, reachable inconsistencies are syntactically represented by the elements of the set Inc (see Definition 4, Section 3.1). Hence, from a syntactic point of view, the semantic approach will falsify as many elements of the set $\mathcal{I} n c$ as possible. So, from this point of view, the semantic approach to modal inconsistency looks exactly the same as the syntactic approach. The only difference seems to be that in the semantic approach, the possibility operators that might occur in the elements of the set $\mathcal{I} n c$ are not considered an integral part of the inconsistency (as in the syntactic approach), but are taken to point to the fact that some inconsistency is true in a reachable world (or, that some reachable world is inconsistent). However, this is not merely a difference in interpretation, because for some premise sets the approaches do yield different consequence sets (see below).

Comparing the Approaches I. To compare both approaches, I will point out the differences and correspondences between the logics $\mathbf{i} \mathbf{A} \mathbf{T} \overline{\mathbf{u}} \mathbf{N} \mathbf{s}_{\mathbf{s y n}}^{\mathbf{r}}$ and $\mathbf{i A T} \overline{\mathbf{u}} \mathbf{N s} \mathbf{s}_{\mathbf{s e m}}^{\mathbf{r}}$, obtained by application of respectively the syntactic and the semantic approach to modal inconsistency.

First of all, inconsistency-adaptive modal logics have the same upper limit logic in case they are characterized by the same lower limit logic, even if they are obtained by application of a different approach to modal inconsistency. This is due to the fact that both approaches falsify as many elements of the set $\mathcal{I} n c$ as possible. Hence, for both approaches the upper limit logic is the logic that interprets the elements of the set Inc as false unconditionally. Obviously, this logic will only be different in case the lower limit logic is different. As a consequence, the upper limit logic of $\mathbf{i A T} \overline{\mathbf{u}} \mathbf{N} \mathbf{s}_{\mathbf{s y n}}^{\mathbf{r}}$ and $\mathbf{i} \mathbf{A T} \overline{\mathbf{u}} \mathbf{N} \mathbf{s}_{\text {sem }}^{\mathbf{r}}$, both based on the lower limit logic $\mathbf{T} \overline{\mathbf{u}} \mathbf{N s}$, is the logic $\mathbf{T}$ (because of Theorem 3 ).

Secondly, for some premise sets, these approaches yield different consequence sets. As this is best illustrated by means of an example, consider the premise set $\Gamma=\{q \vee \diamond(p \wedge \sim p), \diamond \diamond(p \wedge \sim p)\}$. For the logic $\mathbf{i A T} \overline{\mathbf{u}} \mathbf{N s}_{\mathbf{s y n}}^{\mathbf{r}}$, the formula $q$ is derivable from $\Gamma$ in case it is safe to in- 
terpret the triviality-generating formula $\diamond(p \wedge \sim p)$ as false. This is the case, for the formula $\diamond(p \wedge \sim p)$ is not derivable from $\Gamma$ by means of the lower limit logic $\mathbf{T} \overline{\mathbf{u}} \mathbf{N s}$. Hence, the formula $q$ is $\mathbf{i A T} \overline{\mathbf{u}} \mathbf{N} \mathbf{s}_{\mathbf{s y n}}^{\mathbf{r}}$-derivable from $\Gamma$. On the other hand, for the logic $\mathbf{i A T} \overline{\mathbf{u}} \mathbf{N s} \mathbf{s}_{\text {sem }}^{\mathbf{r}}$, the formula $q$ is derivable from $\Gamma$ in case it is safe to state that $p \wedge \sim p$ is not a reachable inconsistency. For, if $p \wedge \sim p$ is not a reachable inconsistency, then the formula $\diamond(p \wedge \sim p)$ has to be false (as it expresses that $p \wedge \sim p$ is a reachable inconsistency). Because of this, the formula $q$ becomes derivable from $\Gamma$. However, the formula $\diamond \diamond(p \wedge \sim p)$ also expresses that $p \wedge \sim p$ is a reachable inconsistency. Hence, as the latter is derivable from $\Gamma$ by means of the lower limit logic $\mathbf{T} \overline{\mathbf{u}} \mathbf{N s}$, it is not safe to state that $p \wedge \sim p$ is not a reachable inconsistency. As a consequence, the formula $q$ is not an $\mathbf{i A T} \overline{\mathbf{u}} \mathbf{N s}_{\text {sem }}^{\mathbf{r}}$-consequence of $\Gamma$.

Finally, the choice for a particular approach to modal inconsistency is a fundamental one, for for some premise sets the approaches yield incomparable consequence sets. By this, I mean that neither of these consequence sets is a subset of the other. (Of course, for some premise sets, the respective consequence sets will be comparable, sometimes even identical). Moreover, irrespective of the specific constraints on the accessibility relation of the modal lower limit logic, there will always be premise sets for which the consequence set yielded by an inconsistency-adaptive modal logic based on the syntactic approach is incomparable with the consequence set yielded by the corresponding inconsistency-adaptive modal logic based on the semantic approach. This will be proven later on (in Section 3.3.3).

\subsection{The inconsistency-adaptive modal logics iATūNss $s_{\text {syn }}^{r}$ and iATūNs sem}

In this section, I will characterize both the semantics and proof theory of the inconsistency-adaptive modal logics iATūNs $\mathbf{s}_{\text {syn }}^{\mathbf{r}}$ and $\mathbf{i A T} \overline{\mathbf{u}} \mathbf{N} \mathbf{s}_{\mathbf{s e m}}^{\mathbf{r}}$. Although both logics are characterized differently, some parts of their characterization are equivalent. For those parts, I will use $\mathbf{i A T} \overline{\mathbf{u} N \mathbf{s}^{\mathbf{r}}}$ in order to refer to both $\mathbf{i A T} \overline{\mathbf{u}} \mathbf{N} \mathbf{s}_{\text {sem }}^{\mathbf{r}}$ and $\mathbf{i} \mathbf{A T} \overline{\mathbf{u}} \mathbf{N} \mathbf{s}_{\mathbf{s y n}}^{\mathbf{r}}$ simultaneously.

\subsubsection{Semantics}

Intuitively, the logic $\mathbf{i A T} \overline{\mathbf{u}} \mathbf{N} \mathbf{s}^{\mathbf{r}}$ interprets a premise set as consistently as possible (see Section 3.2). Semantically, this is expressed by the fact 
that the $\mathbf{i A T} \overline{\mathbf{u}} \mathbf{N} \mathbf{s}^{\mathbf{r}}$-models of a premise set are those $\mathbf{T} \overline{\mathbf{u}} \mathbf{N} \mathbf{s}$-models of a premise set that verify the fewest abnormalities. In other words, the semantics of the logic $\mathbf{i} \mathbf{A} \mathbf{T} \overline{\mathbf{u}} \mathbf{N} \mathbf{s}^{\mathbf{r}}$ is a so-called preferential semantics, meaning that some models of a premise set are preferred over others. As a consequence, the $\mathbf{i A T} \overline{\mathbf{u}} \mathbf{N s}^{\mathbf{r}}$-consequences of a premise set are defined by reference to the set of preferred $\mathbf{T} \overline{\mathbf{u}} \mathbf{N s}$-models of that premise set. Because the logic $\mathbf{i} \mathbf{A T} \overline{\mathbf{u}} \mathbf{N s}^{\mathbf{r}}$ is based on the reliable strategy, this set is the set of the reliable $\mathbf{T} \overline{\mathbf{u}} \mathbf{N}$ s-models of that premise set.

Definition 7. $\Gamma \vDash_{\mathbf{i A T} \mathbf{u} N s^{\mathbf{r}}} A$ ( $A$ is an $\mathbf{i A T} \overline{\mathbf{u}} \mathbf{N} \mathbf{s}^{\mathbf{r}}$-consequence of $\Gamma$ ) iff $A$ is verified by all reliable $\mathbf{T} \overline{\mathbf{u}} \mathbf{N s}$-models of $\Gamma$.

As the reliable models of a premise set $\Gamma$ are determined differently for the logics $\mathbf{i} \mathbf{A T} \overline{\mathbf{u}} \mathbf{N} \mathbf{s}_{\mathbf{s y n}}^{\mathbf{r}}$ and $\mathbf{i} \mathbf{A T} \overline{\mathbf{u}} \mathbf{N} \mathbf{s}_{\text {sem }}^{\mathbf{r}}$, they will be characterized separately.

The Syntactic Approach. Whether a particular TūNs-model $M$ of a premise set $\Gamma$ is also an $\mathbf{i} \mathbf{A} \mathbf{T} \overline{\mathbf{u}} \mathbf{N} \mathbf{s}_{\mathbf{s y n}}^{\mathbf{r}}$-model of $\Gamma$ (henceforth, a reliable ${ }_{\text {syn }}$

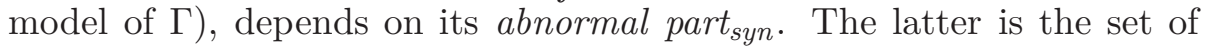
abnormalities $_{\text {syn }}$ that are verified by the model.

Definition 8. Where $M$ is a $\mathbf{T} \overline{\mathbf{u}} \mathbf{N}$ s-model, the abnormal part ${ }_{\text {syn }}$ of $M$ is the set $A b(M)_{\text {syn }}=\left\{A \in \Omega_{\text {syn }} \mid v_{M}\left(A, w_{0}\right)=1\right\}$.

More specifically, in order for a $\mathbf{T} \overline{\mathbf{u}} \mathbf{N s}$-model of a premise set $\Gamma$ to be a reliable ${ }_{\text {syn }}$ model of $\Gamma$, its abnormal part ${ }_{\text {syn }}$ should not contain any abnormalities syn $_{\text {nat }}$ the not also in the set $U(\Gamma)_{s y n}$, the set of unreliable $_{\text {syn }}$ formulas of $\Gamma$.

Definition 9. A TūNs-model $M$ of $\Gamma$ is reliable $s y n$ iff $A b(M)_{s y n} \subseteq$ $U(\Gamma)_{s y n}$.

The set of unreliable ${ }_{s y n}$ formulas of a premise set $\Gamma$ is the set of all abnormalities $_{\text {syn }}$ that occur in a minimal $D a b_{s y n}$-consequence of $\Gamma$. A $D a b_{s y n}$-consequence of $\Gamma$, is a $D a b_{s y n}$-formula (a disjunction of abnormali$\left.\operatorname{ties}_{s y n}\right)$ that is $\mathbf{T} \overline{\mathbf{u}} \mathbf{N}$-derivable from $\Gamma$. Moreover, a minimal $D a b_{s y n^{-}}$ consequence of a premise set $\Gamma$ is a $D a b_{s y n}$-consequence of $\Gamma$ for which

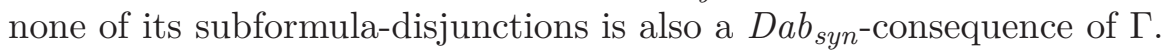

Notational Convention 1. $\operatorname{Dab}(\Delta)=\bigvee(\Delta)$, for $\Delta \subset \Omega_{\text {syn }}$. 
Definition 10. $\operatorname{Dab}(\Delta)$ is a minimal $D a b_{\text {syn-consequence of } \Gamma \text { iff }}$ $\Gamma \vDash_{\mathbf{T} \overline{\mathbf{u} N \mathbf{s}}} \operatorname{Dab}(\Delta)$ and there is no $\Delta^{\prime} \subset \Delta$ such that $\Gamma \vDash_{\mathbf{T} \overline{\mathbf{u} N \mathbf{s}}} \operatorname{Dab}\left(\Delta^{\prime}\right)$.

Definition 11. $U(\Gamma)_{\text {syn }}=\Delta_{1} \cup \Delta_{2} \cup \ldots$ for $\operatorname{Dab}\left(\Delta_{1}\right), \operatorname{Dab}\left(\Delta_{2}\right), \ldots$ the minimal $D a b_{s y n}$-consequences of $\Gamma$.

The Semantic Approach. The iATū $\mathbf{N} \mathbf{s}_{\text {sem }}^{\mathrm{r}}$-models of a premise set (henceforth, the reliable ${ }_{\text {sem }}$ models of a premise set) are determined more

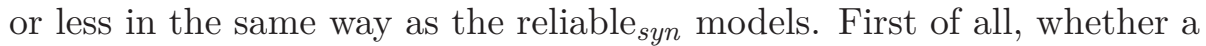

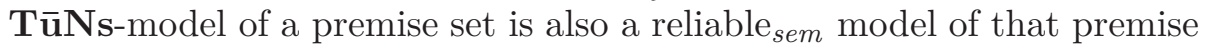
set, depends heavily on the abnormal part ${ }_{\text {sem }}$ of the model, which is the set of all reachable inconsistencies it verifies.

Definition 12. Where $M$ is a $\mathbf{T} \overline{\mathbf{u}} \mathbf{N s}$-model, the set of reachable worlds of $M$ is the set $\operatorname{Reach}(M)=\left\{w_{n} \in W \mid w_{n}=w_{0}\right.$, or $R w_{0} w_{n}$ is the case in $M$, or $\exists w_{i_{1}}, \ldots, w_{i_{n-1}} \in W$ such that $R w_{0} w_{i_{1}}, R w_{i_{1}} w_{i_{2}}, \ldots, R w_{i_{n-1}} w_{n}$ are the case in $M$ \}.

DeFinition 13. Where $M$ is a $\mathbf{T} \overline{\mathbf{u}} \mathbf{N}$ s-model, the abnormal part sem $_{\text {sem }}$ of $M$ is the set $A b(M)_{\text {sem }}=\left\{A \in \Omega_{\text {sem }} \mid \exists w \in \operatorname{Reach}(M): v_{M}(A, w)=1\right\}$.

More specifically, a $\mathbf{T} \overline{\mathbf{u}} \mathbf{N s}$-model of a premise set $\Gamma$ is a reliable sem $_{\text {s. }}$

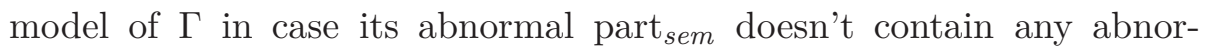
malities $_{\text {sem }}$ (elements of $\Omega_{\text {sem }}$ ) that are not also in the set $U(\Gamma)_{\text {sem }}$, the set of unreliable ${ }_{\text {sem }}$ formulas of $\Gamma$.

Definition 14. A TūNs-model $M$ of $\Gamma$ is reliable sem $_{\text {iff }} A b_{\text {sem }}(M) \subseteq$ $U(\Gamma)_{\text {sem }}$.

The set $U_{\text {sem }}(\Gamma)$ is the set of all abnormalities sem that occur in a minimal $D a b_{\text {sem }}$-consequence of $\Gamma$.

Notational Convention 2. $\operatorname{Dab} b^{\Theta}(\Delta)=\bigvee(\Theta)$, for $\Theta \subset \mathcal{I}$ nc and $\Delta=$ $\left\{A \in \Omega_{\text {sem }} \mid A\right.$ occurs in $\left.\bigvee(\Theta)\right\}$.

Definition 15. $U(\Gamma)_{s e m}=\Delta_{1} \cup \Delta_{2} \cup \ldots$ for $\operatorname{Dab}^{\Theta_{1}}\left(\Delta_{1}\right), \operatorname{Dab}^{\Theta_{2}}\left(\Delta_{2}\right)$, ... the minimal $D a b_{\text {sem }}$-consequences of $\Gamma$.

A $D a b_{s e m}$-consequence of $\Gamma$ is a $D a b_{s e m}$-formula (a disjunction of elements of the set $\mathcal{I} n c$ !) that is $\mathbf{T} \overline{\mathbf{u}} \mathbf{N s}$-derivable from $\Gamma$. Moreover, a minimal $D a b_{\text {sem }}$-consequence $D a b^{\Theta}(\Delta)$ of $\Gamma$ is a $D a b_{\text {sem }}$-consequence of $\Gamma$ for which there is no other $D a b_{\text {sem }}$-consequence $D a b^{\Theta^{\prime}}\left(\Delta^{\prime}\right)$ of $\Gamma$ such that $\Delta^{\prime} \subset \Delta$. 


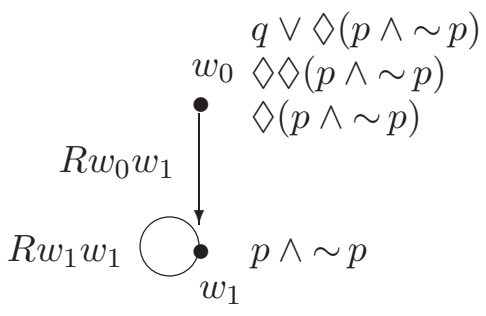

Table 1. A TūNs-model of $\Gamma$.

Definition 16. $\operatorname{Dab}^{\Theta}(\Delta)$ is a minimal $D a b_{s e m}$-consequence of $\Gamma$ iff $\Gamma \vDash_{\text {TūNs }} \operatorname{Dab}^{\Theta}(\Delta)$ and there is no $\Theta^{\prime} \subset \mathcal{I}$ nc and no $\Delta^{\prime} \subset \Delta$ such that also $\Gamma \vDash_{\text {TūNs }} D a b^{\Theta^{\prime}}\left(\Delta^{\prime}\right)$.

Comparing the Approaches II. The difference between the syntactic and the semantic approach to modal inconsistency can now be stated more clearly. Consider again the premise set $\Gamma=\{q \vee \diamond(p \wedge \sim p), \diamond \diamond(p \wedge$ $\sim p)\}$ from Section 3.2. Now, consider both the set of unreliable ${ }_{s y n}$ for-

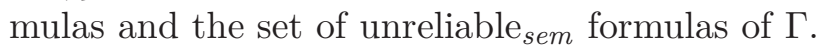

1. $U(\Gamma)_{\text {syn }}=\{\diamond \diamond(p \wedge \sim p), \diamond \diamond \diamond(p \wedge \sim p), \diamond \diamond \diamond \diamond(p \wedge \sim p), \ldots\}$

2. $U(\Gamma)_{\text {sem }}=\{p \wedge \sim p\}$

As a consequence, all reliable ${ }_{\text {syn }}$ models of $\Gamma$ falsify the formula $\diamond(p \wedge$ $\sim p$ ) (as it is not an element of $U(\Gamma)_{\text {syn }}$ ). Hence, the formula $q$ is an iAT $\overline{\mathbf{u}} \mathbf{N s}_{\mathbf{s y n}}^{\mathbf{r}}$-consequence of $\Gamma$ (for it is verified by all reliable ${ }_{\text {syn }}$ models

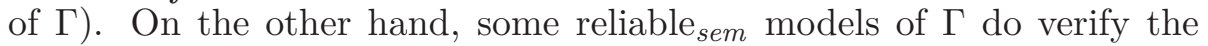
formula $\diamond(p \wedge \sim p)$ (for example, see table 1 ). As some of these models also falsify the formula $q, q$ is not an $\mathbf{i A T} \overline{\mathbf{u}} \mathbf{N s}_{\mathbf{s e m}}^{\mathbf{r}}$-consequence of $\Gamma$.

\subsubsection{Proof Theory}

As $\mathbf{i A T} \overline{\mathbf{u}} \mathbf{N s}^{\mathbf{r}}$ is an adaptive logic, its proof theory has some characteristic features that are shared by all adaptive logics. First of all, an iATūNs ${ }^{\mathbf{r}}$-proof is a succession of stages, each consisting of a sequence of lines. Adding a line to a proof means to move on to a next stage of the proof. Secondly, the lines of an $\mathbf{i A T} \overline{\mathbf{u}} \mathbf{N} \mathbf{s}^{\mathbf{r}}$-proof consist of four elements (instead of the usual three): a line number, a formula, a justification, 
and an adaptive condition. The latter is a finite subset of $\Omega$ (the set of abnormalities). Thirdly, as long as all elements of the adaptive condition of a line $i$ can be considered as false, the formula of line $i$ is considered as derivable from the premise set. In order to indicate that not all elements of the adaptive condition of line $i$ can be considered as false anymore, line $i$ is marked (formally, this is done by placing the symbol $\checkmark$ next to the adaptive condition). Obviously, when a line is marked, the formula on that line is not considered as derivable anymore. Finally, the markings of an $\mathbf{i A T} \mathbf{\mathbf { u }} \mathbf{N s}^{\mathbf{r}}$-proof are dynamic. At some stage of the proof, a line might be marked (resp. unmarked), while at a later stage, it might become unmarked (resp. marked) again.

General Characterization. The $\mathbf{i A T} \overline{\mathbf{u}} \mathbf{N} \mathbf{s}^{\mathbf{r}}$-proof theory consists of both deduction rules and a marking criterion. The deduction rules determine how new lines may be added to a proof, while the marking criterion determines at every stage of the proof which lines have to be marked.

As the deduction rules and the marking criterion are different for the $\operatorname{logics} \mathbf{i} \mathbf{A T} \overline{\mathbf{u}} \mathbf{N} \mathbf{s}_{\mathbf{s y n}}^{\mathbf{r}}$ and $\mathbf{i} \mathbf{A} \mathbf{T} \overline{\mathbf{u}} \mathbf{N} \mathbf{s}_{\text {sem }}^{\mathbf{r}}$, they will be characterized separately.

The Syntactic Approach. The deduction rules of the logic iAT $\overline{\mathbf{u}} \mathbf{N} \mathbf{s}_{\mathbf{s y n}}^{\mathbf{r}}$ are listed in shorthand notation, with

\section{$A \quad \Delta$}

expressing that $A$ occurs in the proof on the condition $\Delta$.

PREM If $A \in \Gamma$ :

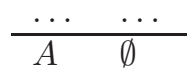

RU

If $A_{1}, \ldots, A_{n} \vdash_{\mathbf{T u} \overline{\mathbf{N} s}} B$ :

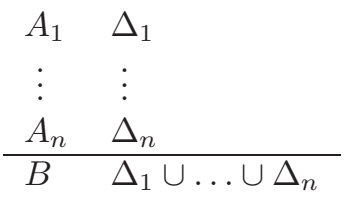

$\mathrm{RC}_{\text {syn }}$

$$
\begin{aligned}
& \text { If } A_{1}, \ldots, A_{n} \vdash_{\mathbf{T u} \text { us }} B \vee \operatorname{Dab}(\Theta) \quad A_{1} \quad \Delta_{1} \\
& \begin{array}{ll}
A_{n} & \Delta_{n} \\
\hline B & \Delta_{1} \cup \ldots \cup \Delta_{n} \cup \Theta
\end{array}
\end{aligned}
$$


Notice that the deduction rules are fully determined by the logic $\mathbf{T} \overline{\mathbf{u}} \mathbf{N} \mathbf{s}$ (the lower limit logic of $\mathbf{i A T} \overline{\mathbf{u}} \mathbf{N} \mathbf{s}_{\mathbf{s y n}}^{\mathbf{r}}$ ) and by the set of abnormalities $\Omega_{\text {syn }}$. Hence, the deduction rules are completely independent of the adaptive strategy. On the other hand, the marking criterion is completely determined by the adaptive strategy, in this case the reliability strategy.

Whether a line $i$ is marked at a stage $s$ of an $\mathbf{i A T} \overline{\mathbf{u}} \mathbf{N} \mathbf{s}_{\mathbf{s y n}}^{\mathbf{r}}-$ proof, depends on the set $U_{s}(\Gamma)_{s y n}$, the set of unreliable syn $_{\text {formulas at stage }}$ $s$ of the proof. The latter are the abnormalities syn that occur in the minimal $D a b_{s y n}$-consequences that have been derived at stage $s$.

Definition 17. $\operatorname{Dab}(\Delta)$ is a minimal $D a b_{s y n}$-consequence of $\Gamma$ at stage $s$ of the proof iff $\operatorname{Dab}(\Delta)$ is derived at stage $s$ on the condition $\emptyset$ and there is no $\Delta^{\prime} \subset \Delta$ such that $\operatorname{Dab}\left(\Delta^{\prime}\right)$ is also derived at stage $s$ on the condition $\emptyset$.

Definition 18. $U_{s}(\Gamma)_{\text {syn }}=\Delta_{1} \cup \Delta_{2} \cup \ldots$ for $\operatorname{Dab}\left(\Delta_{1}\right), \operatorname{Dab}\left(\Delta_{2}\right), \ldots$ the minimal $D a b_{s y n}$-consequences of $\Gamma$ at stage $s$ of the proof.

Marking now proceeds as follows: at a stage $s$, a line is marked in case its condition shares some abnormalities with the set $U_{s}(\Gamma)_{s y n}$. Hence, consider the actual marking definition of the logic $\mathbf{i A T} \overline{\mathbf{u}} \mathbf{N} \mathbf{s}_{\mathbf{s y n}}^{\mathbf{r}}$ :

Definition 19. Line $i$ is marked at stage $s$ iff, where $\Delta$ is its condition, $\Delta \cap U_{s}(\Gamma)_{s y n} \neq \emptyset$.

The Semantic Approach. The logic $\mathbf{i A T} \overline{\mathbf{u}} \mathbf{N s}_{\text {sem }}^{\mathbf{r}}$ also has three deduction rules: PREM, RU, and $\mathrm{RC}_{\text {sem. }}$. The rules PREM and RU are as for the logic $\mathbf{i} \mathbf{A T} \overline{\mathbf{u}} \mathbf{N s}_{\text {syn }}^{\mathbf{r}}$ (see above). Hence, only the rule $\mathrm{RC}_{\text {sem }}$ is new. Consider it below.

$$
\begin{array}{lll}
\mathrm{RC}_{\text {sem }} \text { If } A_{1}, \ldots, A_{n} \vdash{ }_{\mathbf{T u} \mathbf{u} \mathbf{s}} B \vee \operatorname{Dab}^{\Theta}(\Sigma) & A_{1} & \Delta_{1} \\
& \vdots & \vdots \\
& A_{n} & \Delta_{n} \\
\hline & B & \Delta_{1} \cup \ldots \cup \Delta_{n} \cup \Sigma
\end{array}
$$

Not only the deduction rules of the logic iAT $\mathbf{i} \mathbf{N} \mathbf{s s}_{\text {sem }}^{\mathbf{r}}$ are quite similar to those of the logic $\mathbf{i} \mathbf{A} \mathbf{T} \overline{\mathbf{u}} \mathbf{N} \mathbf{s}_{\mathbf{s y n}}^{\mathbf{r}}$, also the marking criterion is. More specifically, marking for $\mathbf{i} \mathbf{A T} \overline{\mathbf{u}} \mathbf{N} \mathbf{s}_{\text {sem }}^{\mathbf{r}}$ is defined by reference to the

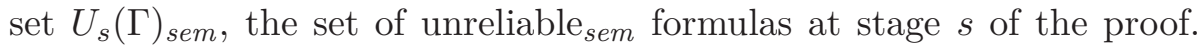
The latter are the abnormalities ${ }_{s e m}$ that occur in the minimal $D a b_{s e m}{ }^{-}$ consequences that have been derived at stage $s$. 
Definition 20. $\operatorname{Dab}^{\Theta}(\Delta)$ is a minimal $D_{a b} b_{\text {sem }}$-consequence of $\Gamma$ at stage $s$ of the proof iff $D a b^{\Theta}(\Delta)$ has been derived at stage $s$ on the condition $\emptyset$, and there is no $\Theta^{\prime} \subset \mathcal{I} n c$ and no $\Delta^{\prime} \subset \Delta$ such that $D a b^{\Theta^{\prime}}\left(\Delta^{\prime}\right)$ is also derived at stage $s$ on condition $\emptyset$.

Definition 21. $U_{s}(\Gamma)_{\text {sem }}=\Delta_{1} \cup \Delta_{2} \cup \ldots$ for $\operatorname{Dab}^{\Theta_{1}}\left(\Delta_{1}\right), \operatorname{Dab}^{\Theta_{2}}\left(\Delta_{2}\right)$, ... the minimal $D a b_{\text {sem }}$-consequences of $\Gamma$ at stage $s$ of the proof.

Marking for the logic iAT $\mathbf{i} \mathbf{N} \mathbf{s}_{\text {sem }}^{\mathbf{r}}$ now proceeds as follows: at stage $s$, a line is marked in case its condition shares some abnormalities sem $_{\text {s }}$ with the set $U_{s}(\Gamma)_{\text {sem }}$. Hence, consider the actual marking definition:

Definition 22. Line $i$ is marked at stage $s$ iff, where $\Delta$ is its condition, $\Delta \cap U_{s}(\Gamma)_{\text {sem }} \neq \emptyset$.

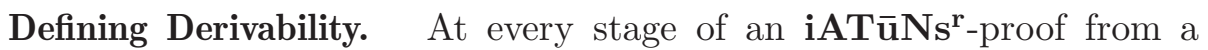
premise set $\Gamma$, all formulas that occur as the second element of an unmarked line are considered as derivable from $\Gamma$.

Definition 23. $A$ is derived from $\Gamma$ at stage $s$ of an $\mathbf{i A T} \overline{\mathbf{u}} \mathbf{N} \mathbf{s}^{\mathbf{r}}$-proof iff $A$ is the second element of an unmarked line at stage $s$.

However, because of the dynamic nature of $\mathbf{i A T} \overline{\mathbf{u}} \mathbf{N} \mathbf{s}^{\mathbf{r}}$-proofs, this definition of derivability is rather problematic. Markings may change at every stage of the proof, so that for every new stage, it has to be reconsidered whether or not a formula is derivable from the premise set. Luckily, it is also possible to define a stable notion of derivability, called final derivability.

Definition 24. $A$ is finally derived from $\Gamma$ on line $i$ of a proof at stage $s$ iff (i) $A$ is the second element of line $i$, (ii) line $i$ is not marked at stage $s$, and (iii) every extension of the proof in which line $i$ is marked may be further extended in such a way that line $i$ is unmarked.

Because of its stability, the notion of final derivability is used to define iAT $\overline{\mathbf{u}} \mathbf{N s}^{\mathrm{r}}$-derivability.

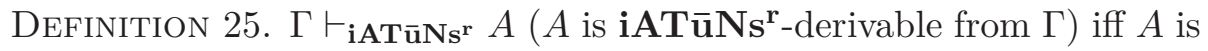
finally derived on a line of a proof from $\Gamma$. 
Example. In order to illustrate the proof theory of both iAT $\mathbf{i} \mathbf{N} \mathbf{s}_{\text {syn }}^{\mathbf{r}}$ and $\mathbf{i A T} \overline{\mathbf{u}} \mathbf{N s}_{\text {sem }}^{\mathbf{r}}$, consider the proof below. It is based on the premise set $\Gamma=\{\square(q \vee p), \diamond \square \sim p, \square(p \vee r), \square \square \sim r, \diamond r, \square \diamond p\}$. In order to use the same example for both logics, each line of the proof below has two adaptive conditions. The left one is the $\mathbf{i A T} \overline{\mathbf{u}} \mathbf{N} \mathbf{s}_{\mathbf{s y n}}^{\mathbf{r}}$-condition, while the right one is the $\mathbf{i A T} \overline{\mathbf{u}} \mathbf{N s} \mathbf{s e m}_{\mathbf{s e m}}^{\mathbf{r}}$-condition. A line is marked for the logic iAT $\overline{\mathbf{u}} \mathbf{N s}_{\text {syn }}^{\mathbf{r}}\left(\right.$ resp. iAT $\left.\overline{\mathbf{u}} \mathbf{N s}_{\text {sem }}^{\mathbf{r}}\right)$ in case a $\checkmark$-symbol is placed next to the corresponding condition.
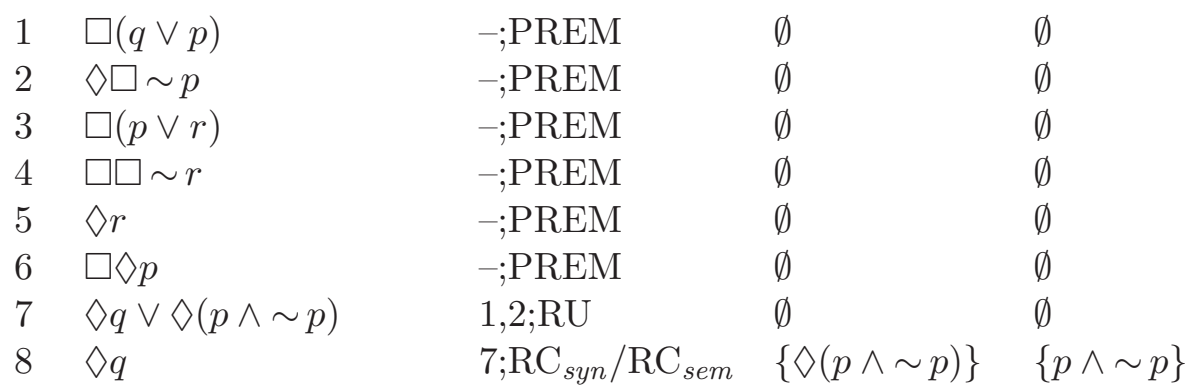

At stage 8 of the proof, no $D a b$-consequences have been derived yet. As a consequence, both $U_{8}(\Gamma)_{s y n}$ and $U_{8}(\Gamma)_{\text {sem }}$ are empty. Accordingly, no lines are marked, so that the formula $\diamond q$ (on line 8) is considered as derivable from $\Gamma$ by both iATūNs $\mathbf{s}_{\mathbf{s y n}}^{\mathbf{r}}$ and $\mathbf{i} \mathbf{A T} \overline{\mathbf{u}} \mathbf{N s}_{\text {sem }}^{\mathbf{r}}$.
$8 \diamond q$
$9 \diamond(p \wedge \sim p) \vee \diamond(r \wedge \sim r) 2,3,4 ; \mathrm{RU}$
$7 ; \mathrm{RC}_{\text {syn }} / \mathrm{RC}_{\text {sem }}$
$\underset{\emptyset}{\{\oslash(p \wedge \sim p)\}} \underset{\emptyset}{\{p \wedge \sim p\} \checkmark}$

At stage 9 of the proof, a $D a b$-consequence has been derived on line 9 . As a consequence, $U_{9}(\Gamma)_{s y n}=\{\diamond(p \wedge \sim p), \diamond(r \wedge \sim r)\}$ and $U_{9}(\Gamma)_{s e m}=\{p \wedge$ $\sim p, r \wedge \sim r\}$, which results in the marking of line 8 for both $\mathbf{i A T} \overline{\mathbf{u}} \mathbf{N} \mathbf{s}_{\mathbf{s y n}}^{\mathbf{r}}$ and $\mathbf{i A T} \overline{\mathbf{u}} \mathbf{N s}_{\text {sem }}^{\mathbf{r}}$. Hence, at stage 9 of the proof, the formula $\diamond q$ is not considered as derivable anymore.

$\begin{array}{lllll}8 & \nabla q & 7 ; \mathrm{RC}_{\text {syn }} / \mathrm{RC}_{\text {sem }} & \{\diamond(p \wedge \sim p)\} & \{p \wedge \sim p\} \\ 9 & \vee(p \wedge \sim p) \vee \diamond(r \wedge \sim r) & 2,3,4 ; \mathrm{RU} & \emptyset & \emptyset \\ 10 \diamond(r \wedge \sim r) & 4,5 ; \mathrm{RU} & \emptyset & \emptyset\end{array}$

At stage 10 of the proof, the $D a b$-consequence on line 9 is not a minimal $D a b$-consequence anymore. For, on line 10 a smaller $D a b$-consequence has been derived. As a consequence, $U_{10}(\Gamma)_{\text {syn }}=\{\oslash(r \wedge \sim r)\}$ and 
$U_{10}(\Gamma)_{\text {sem }}=\{r \wedge \sim r\}$. Hence, line 8 becomes unmarked again, so that the formula $\diamond q$ is again considered as derivable from $\Gamma$.

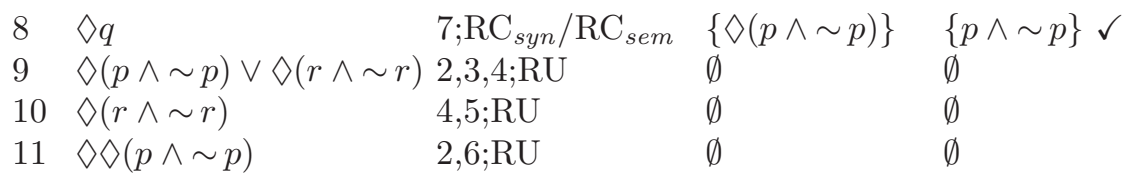

Finally, at stage 11 of the proof, another $D a b$-consequence has been derived. As a consequence, $U_{11}(\Gamma)_{\text {syn }}=\{\diamond(r \wedge \sim r), \diamond \diamond(p \wedge \sim p)\}$ and $U_{11}(\Gamma)_{\text {sem }}=\{r \wedge \sim r, p \wedge \sim p\}$. Line 8 becomes marked again, but only for the logic $\mathbf{i A T} \overline{\mathbf{u}} \mathbf{N} \mathbf{s}_{\text {sem }}^{\mathbf{r}}$. Hence, the formula $\diamond q$ is now considered as derivable from $\Gamma$ by means of the logic $\mathbf{i A T} \mathbf{\mathbf { u }} \mathbf{N s}_{\mathbf{s y n}}^{\mathbf{r}}$, but not by means of the logic $\mathbf{i A T} \overline{\mathbf{u}} \mathbf{N} \mathbf{s}_{\mathbf{s e m}}^{\mathbf{r}}$. Moreover, given the premise set $\Gamma$, this will also be the case at the final stage of the proof.

\subsubsection{Metatheory}

First, I will prove soundness and completeness for both iATū $\mathbf{i} \mathbf{s}_{\mathbf{s y n}}^{\mathbf{r}}$

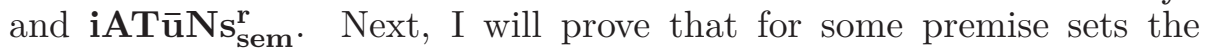
approaches to modal inconsistency yield incomparable consequence sets (as was stated in Section 3.2).

Soundness and Completeness. The logic iAT $\overline{\mathbf{u}} \mathbf{N} \mathbf{s}_{\mathbf{s y n}}^{\mathbf{r}}$ is characterized according to the standard format of adaptive logics (see Section 2). Hence, as soundness and completeness were proven for all adaptive logics that are characterized accordingly (see [5]), soundness and completeness for the logic iATū $\mathbf{i} \mathbf{s}_{\mathbf{s y n}}^{\mathrm{r}}$ are given.

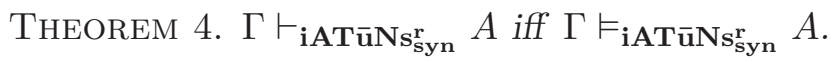

The logic $\mathbf{i A T} \overline{\mathbf{u}} \mathbf{N s} \mathbf{s}_{\mathbf{s e m}}^{\mathbf{r}}$ is not characterized according to the standard format. At least, not completely. Hence, soundness and completeness of $\mathbf{i A T} \overline{\mathbf{u}} \mathbf{N} s_{\text {sem }}^{\mathbf{r}}$ are proven below. First, consider the theorem below.

TheOREM 5. $\Gamma \vdash_{\mathrm{iATu} \mathbf{A} \mathbf{s}_{\mathrm{sem}}^{\mathrm{r}}} A$ iff for some finite $\Theta \subset \mathcal{I}$ c and $\Delta \subset \Omega_{\text {sem }}$, $\Gamma \vdash_{\mathbf{T u} N \mathbf{u}} A \vee \operatorname{Dab}^{\Theta}(\Delta)$ and $\Delta \cap U(\Gamma)_{\text {sem }}=\emptyset$.

Proof. The proof is completely equivalent to the proof of Theorem 6 in $[5$, p. 233]. Hence, it is left to the reader. 
Next, some preliminary remarks are necessary. First, for each set $\Delta \subseteq \mathcal{W}$, there is a set $\Delta^{\diamond}$ that is defined as follows:

Definition 26. $\Delta^{\diamond}=\Delta \cup\left\{\diamond \ldots \diamond(A) \in \mathcal{W}^{\mathcal{M}} \mid A \in \Delta\right\}$.

Second, in the proofs below, I will make use of the classical negation $\neg$. The latter will be used in a metatheoretic way, to express that a formula is interpreted as false in the classical sense (hence, the formula cannot be both true and false at the same time). As a consequence, it is possible for each set $\Delta$ to define a set $\Delta\urcorner$.

Definition 27. $\Delta\urcorner=\{\neg A \mid A \in \Delta\}$.

Now, the following preliminary lemmas can easily be proven by relying on Definition 26.

Lemma 1. For all TūNs-models $M, A b(M)_{\text {syn }} \subseteq\left(A b(M)_{\text {sem }}\right)^{\diamond}$.

Proof. Suppose that $A \in A b(M)_{\text {syn }}$. Hence, $M$ verifies $A$. Moreover, as $A$ is of the form $B \wedge \sim B$ or $\diamond \ldots \diamond(B \wedge \sim B$ ) (for $B \in \mathcal{S}), B \wedge \sim B$ is true at some world $w \in \operatorname{Reach}(M)$ (by Definition 12 and the semantic characterization of $\mathbf{T} \overline{\mathbf{u}} \mathbf{N s})$. As a consequence, $B \wedge \sim B \in A b(M)_{\text {sem }}$ (by Definition 13). Of course, this implies that $A \in\left(A b(M)_{\text {sem }}\right)^{\diamond}$ (by Definition 26).

Lemma 2. For all TūNs-models $M$ of a premise set $\Gamma$, if $A b(M)_{\text {sem }} \subseteq$ $U(\Gamma)_{\text {sem }}$ then $\left(A b(M)_{\text {sem }}\right)^{\diamond} \subseteq\left(U(\Gamma)_{\text {sem }}\right)^{\diamond}$.

Proof. Suppose that $A b(M)_{\text {sem }} \subseteq U(\Gamma)_{\text {sem }}$ and that $A \in\left(A b(M)_{\text {sem }}\right)^{\diamond}$. Hence, $A$ is of the form $B \wedge \sim B$ or $\diamond \ldots \diamond(B \wedge \sim B)$, for $B \in \mathcal{S}$ (by definitions 13 and 26). As a consequence, $B \wedge \sim B \in A b(M)_{\text {sem }}$ (by Definition 26). In view of the main supposition, this implies that $B \wedge$ $\sim B \in U(\Gamma)_{\text {sem }}$. Hence, $A \in\left(U(\Gamma)_{\text {sem }}\right)^{\diamond}$ (by Definition 26).

Lemma 3. For all TūNs-models $M$, if $A \in A b(M)_{\text {sem }}$ then there is some $B \in(\{A\})^{\diamond}$ such that $B \in A b(M)_{\text {syn }}$.

Proof. Suppose that $A \in A b(M)_{\text {sem }}$. In view of the semantic characterization of $\mathbf{T} \overline{\mathbf{u}} \mathbf{N s}$, and definitions 12 and 13, this implies that some $B \in(\{A\})^{\diamond}$ is verified by $M$. As a consequence, $B \in A b(M)_{\text {syn }}$ (by Definition 8). 
Lemma 4. For all TūNs-models $M$ of a premise set $\Gamma, A b(M)_{\text {sem }} \subseteq$ $U(\Gamma)_{\text {sem }}$ iff $A b(M)_{\text {syn }} \subseteq\left(U(\Gamma)_{\text {sem }}\right)^{\diamond}$.

Proof. Left-Right. Suppose that $A b(M)_{\text {sem }} \subseteq U(\Gamma)_{\text {sem }}$ and that $A \in$ $A b(M)_{\text {syn }}$. In view of the lemmas 1 and 2 , these suppositions imply that $A \in\left(U(\Gamma)_{\text {sem }}\right)^{\diamond}$.

Right-Left. Suppose that $A b(M)_{\text {syn }} \subseteq\left(U(\Gamma)_{\text {sem }}\right)^{\diamond}$ and that $A \in$ $A b(M)_{\text {sem. }}$. In view of Lemma 3, these suppositions imply that there is some $B \in(\{A\})^{\diamond}$ such that $B \in\left(U(\Gamma)_{\text {sem }}\right)^{\diamond}$. Well now, suppose that $A \notin U(\Gamma)_{\text {sem }}$. Hence, no $B \in(\{A\})^{\diamond}$ can be such that $B \in\left(U(\Gamma)_{\text {sem }}\right)^{\diamond}$ (by Definition 26). As this contradicts the foregoing, $A \in U(\Gamma)_{\text {sem }}$. $\dashv$

By relying on the lemmas above, the following theorems can now be proven.

Theorem 6. If $\Gamma$ has $\mathbf{T} \overline{\mathbf{u}} \mathbf{N s - m o d e l s ,} \Gamma$ has iAT $\overline{\mathbf{u}} \mathbf{N} \mathbf{s}_{\mathbf{s e m}}^{\mathbf{r}}$-models as well.

Proof. Suppose the set of $\mathbf{T} \overline{\mathbf{u}} \mathbf{N s}$-models of $\Gamma$ is non-empty. Well now, the reliable sem $_{\text {models of }} \Gamma$ are the TüNs-models $M$ of $\Gamma$ for which $A b(M)_{\text {sem }} \subseteq U(\Gamma)_{\text {sem }}$ (see Definition 14). Hence, the reliable sem $_{\text {sedels }}$ of $\Gamma$ are the TūNs-models $M$ of $\Gamma$ for which $A b(M)_{\text {syn }} \subseteq\left(U(\Gamma)_{\text {sem }}\right)^{\diamond}$ (because of Lemma 4). Hence, the reliable ${ }_{\text {sem }}$ models of $\Gamma$ are the $\mathbf{T} \overline{\mathbf{u}} \mathbf{N} \mathbf{s}-$ models of $\Gamma$ that falsify all elements of the set $\left(\left(\Omega_{\text {sem }}\right)^{\diamond}-\left(U(\Gamma)_{\text {sem }}\right)^{\diamond}\right)$. Differently put, the reliable sem $_{\text {models of }} \Gamma$ are the $\mathbf{T} \overline{\mathbf{u}} \mathbf{N}$ s-models of $\left.\Gamma \cup\left(\left(\Omega_{\text {sem }}\right)^{\diamond}-\left(U(\Gamma)_{\text {sem }}\right)^{\diamond}\right)\right\urcorner$. As a consequence, $\Gamma$ has reliable sem $_{\text {sedels }}$ in case $\left.\Gamma \cup\left(\left(\Omega_{\text {sem }}\right)^{\diamond}-\left(U(\Gamma)_{\text {sem }}\right)^{\diamond}\right)\right\urcorner$ has $\mathbf{T} \overline{\mathbf{u}} \mathbf{N}$ s-models. Suppose the latter is not the case. Because $\Gamma$ has $\mathbf{T} \overline{\mathbf{u}} \mathbf{N s}$-models, this is only possible in case there is a finite $\Theta \subset\left(\left(\Omega_{\text {sem }}\right)^{\diamond}-\left(U(\Gamma)_{\text {sem }}\right)^{\diamond}\right)$ such that $\Gamma \vDash_{\text {TūNs }}$ $D a b^{\Theta}(\Delta)$ and for which there are no $\Theta^{\prime} \subset\left(\Omega_{\text {sem }}\right)^{\diamond}, \Delta^{\prime} \subset \Delta$ such that also $\Gamma \vDash_{\mathbf{T} \overline{\mathbf{u}} \mathbf{s}} \operatorname{Dab}^{\Theta^{\prime}}\left(\Delta^{\prime}\right)$. In fact, this means that $\operatorname{Dab}^{\Theta}(\Delta)$ is a minimal $D a b_{\text {sem }}$-consequence of $\Gamma$ (by Definition 16). Hence, $\Delta \subseteq U(\Gamma)_{\text {sem }}$ and $\Theta \subset\left(U(\Gamma)_{\text {sem }}\right)^{\diamond}$. However, because of the construction of $\Theta$, the latter is impossible. As a consequence, $\Gamma$ has reliable ${ }_{\text {sem }}$ models.

TheOREM 7. $\Gamma \vDash_{\text {iATūNs }}$ sem $_{\text {rem }} A$ iff some finite $\Theta \subset \mathcal{I}$ nc and $\Delta \subset \Omega_{\text {sem }}$, $\Gamma \vDash_{\mathbf{T u} \text { ùs }} A \vee D a b^{\Theta}(\Delta)$ and $\Delta \cap U(\Gamma)_{\text {sem }}=\emptyset$.

Proof. Left-Right. Suppose $\Gamma \vDash_{\mathbf{i} A T \bar{u} \mathbf{N s}_{\mathbf{s e m}}^{\mathbf{r}}} A$. Hence, $A$ is verified by all reliable sem $_{\text {models of } \Gamma \text { (by Definition } 7 \text { ). For all reliable }}$ sem models of $\Gamma, A b(M)_{\text {sem }} \subseteq U(\Gamma)_{\text {sem }}$ (by Definition 14). However, this 
also means that all reliable sem $_{\text {models of }} \Gamma$ falsify all elements of the set $\left(\left(\Omega_{\text {sem }}\right)^{\diamond}-\left(U(\Gamma)_{\text {sem }}\right)^{\diamond}\right)$ (by the same reasoning as in the proof of Theorem 6). Differently put, $\left.\Gamma \cup\left(\left(\Omega_{\text {sem }}\right)^{\diamond}-\left(U(\Gamma)_{\text {sem }}\right)^{\diamond}\right)\right\urcorner \vDash_{\text {TūNs }} A$. Moreover, as $\mathbf{T} \overline{\mathbf{u}} \mathbf{N s}$ is compact (easily verified by relying on Definition 3), this implies that there is a finite $\Gamma^{\prime} \subseteq \Gamma$ and a finite $\Theta \subset$ $\left(\left(\Omega_{\text {sem }}\right)^{\diamond}-\left(U(\Gamma)_{\text {sem }}\right)^{\diamond}\right)$ such that $\Gamma^{\prime} \cup \Theta^{\urcorner} \vDash_{\text {TūNs }} A$. But, then also $\Gamma^{\prime} \vDash_{\text {TũNs }} A \vee \bigvee(\Theta)$ (by the characterization of classical negation). Hence, as the logic $\mathbf{T} \overline{\mathbf{u}} \mathbf{N s}$ is monotonic, $\Gamma \vDash_{\mathbf{T}} \mathbf{u} \mathbf{N s}_{\mathbf{s}} A \bigvee \bigvee(\Theta)$, which actually comes down to the following: $\Gamma \vDash_{\text {TūNs }} A \vee D a b^{\Theta}(\Delta)$ for a finite $\Theta \subset \mathcal{I}$ nc and $\Delta \subset \Omega_{\text {sem }}$. Moreover, $\Delta \cap U(\Gamma)_{\text {sem }}=\emptyset$ (otherwise, it is impossible that $\left.\Theta \subset\left(\left(\Omega_{\text {sem }}\right)^{\diamond}-\left(U(\Gamma)_{\text {sem }}\right)^{\diamond}\right)\right)$.

Right-Left. Suppose there is a finite $\Theta \subset \mathcal{I} n c$ and $\Delta \subset \Omega_{\text {sem }}$ such that $\Gamma \vDash_{\text {TūNs }} A \vee D a b^{\Theta}(\Delta)$ and $\Delta \cap U(\Gamma)_{\text {sem }}=\emptyset$. First of all, $\Gamma \vDash_{\mathbf{i} A \mathbf{T} \bar{u} \mathbf{u s}_{\text {sem }}^{\mathrm{r}}} A$ holds vacuously if the set of $\mathbf{T} \overline{\mathbf{u}} \mathbf{N s}$-models is empty. So, suppose it is not empty and that all its members verify $A \vee D a b^{\Theta}(\Delta)$. Hence,

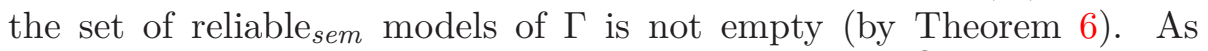
$\Delta \cap U(\Gamma)_{\text {sem }}=\emptyset$, all reliable ${ }_{\text {sem }}$ models falsify $\operatorname{Dab}^{\Theta}(\Delta)$ (otherwise, $\left.A b(M)_{\text {sem }} \nsubseteq U(\Gamma)_{\text {sem }}\right)$. So, all reliable sem models verify $A$. Hence, $\Gamma \vDash_{\text {iATūNs }}{ }^{\mathbf{r}} A$ (by Definition 7 ).

Finally, soundness and completeness for the logic iATūNs $\mathbf{s}_{\text {sem }}^{\mathbf{r}}$ can now be proven.

Corollary 1. $\Gamma \vdash_{\mathbf{i A T u} \mathbf{u} \mathbf{s}_{\mathbf{s e m}}^{\mathrm{r}}} A$ iff $\Gamma \vdash_{\mathbf{i} A T \overline{\mathbf{u}} \mathbf{N s}_{\mathbf{s e m}}^{\mathrm{r}}} A$.

Proof. Immediate from Theorem 5, Theorem 7, and the soundness and completeness of the Logic $\mathbf{T} \overline{\mathbf{u}} \mathbf{N}$ s (Theorem 2).

Comparing the Approaches III. In Section 3.2, I claimed that for some premise sets the syntactic and semantic approach to modal inconsistency yield incomparable consequence sets. Moreover, I claimed they do so irrespective of the specific constrains on the accessibility relation. I will now prove this. First, consider Lemma 5 stating that for some premise sets, the consequence set yielded by inconsistency-adaptive modal logics based on the syntactic approach $\left(\mathbf{i} \mathbf{A M L} \mathbf{L}_{\text {syn }}\right)$ is not a subset of the consequence set yielded by the corresponding inconsistency-adaptive modal logics based on the semantic approach (iAML $\left.\mathbf{i}_{\text {sem }}\right)$.

Lemma 5. For some $\Gamma \subset \mathcal{W}^{\mathcal{M}}$ and some $A \in \mathcal{W}^{\mathcal{M}}, \Gamma \vdash_{\mathbf{i A M L}_{\text {syn }}} A$ and $\Gamma \nvdash_{\mathbf{i A M L}_{\text {sem }}} A$. 
Proof. Consider the premise set $\Gamma=\{q \vee(p \wedge \sim p), \diamond(p \wedge \sim p)\}$. It is easily verified that $p \wedge \sim p \notin U(\Gamma)_{\text {syn }}$, while $p \wedge \sim p \in U(\Gamma)_{\text {sem }}$ moreover, remark that this is the case irrespective of the constraints on the accessibility relation of the modal lower limit logic. As a consequence, the formula $q$ is finally derivable from $\Gamma$ by all $\mathbf{i} \mathbf{A} \mathbf{M L} \mathbf{L}_{\text {syn }}$, but not by any of the corresponding $\mathbf{i} \mathbf{A M L} \mathbf{L}_{\text {sem }}$.

Also consider Lemma 6. This lemma states that for some premise sets, the consequence set yielded by $\mathbf{i} \mathbf{A} \mathbf{M L} \mathbf{L}_{\text {sem }}$ is not a subset of the consequence set yielded by the corresponding $\mathbf{i} \mathbf{A M L} \mathbf{L}_{\text {syn }}$.

Lemma 6. For some $\Gamma \subset \mathcal{W}^{\mathcal{M}}$ and some $A \in \mathcal{W}^{\mathcal{M}}, \Gamma \vdash_{\mathbf{i A M L}_{\text {sem }}} A$ and $\Gamma \nvdash_{\mathbf{i A M L}_{s y n}} A$.

Proof. Consider the premise set $\Gamma=\{r \vee(q \wedge \sim q),(p \wedge \sim p) \vee(q \wedge$ $\sim q), \diamond(p \wedge \sim p)\}$. It is easily verified that $q \wedge \sim q \notin U(\Gamma)_{\text {sem }}$, while $q \wedge \sim q \in U(\Gamma)_{\text {syn }}$ - again, remark that this is the case irrespective of the constraints on the accessibility relation of the modal lower limit logic. Consequently, the formula $r$ is finally derivable from $\Gamma$ by all $\mathbf{i} \mathbf{A} \mathbf{M} \mathbf{L}_{\text {sem }}$, but not by any of the corresponding $\mathbf{i} \mathbf{A M L}_{\text {syn }}$.

Finally, from lemmas 5 and 6 immediately follows that, for some premise sets, $\mathbf{i} \mathbf{A} \mathbf{M L} \mathbf{L}_{\text {syn }}$ and $\mathbf{i} \mathbf{A} \mathbf{M L} \mathbf{L}_{\text {sem }}$ yield incomparable consequence sets.

THEOREM 8. For some $\Gamma \subset \mathcal{W}^{\mathcal{M}}, C n_{\mathbf{i A M L}_{s y n}}(\Gamma) \nsubseteq C n_{\mathbf{i A M L}_{\text {sem }}}(\Gamma)$ and $C n_{\mathbf{A A M L}_{s e m}}(\Gamma) \nsubseteq C n_{\mathbf{i A M L}_{s y n}}(\Gamma)$.

\subsection{A Peculiar Feature: Semi-Explosion}

Despite the many nice features of the inconsistency-adaptive modal framework presented in this paper, some of the inconsistency-adaptive modal logics (iaML) that fall within the framework, do not avoid explosion completely. I am referring to the iAML of which the lower limit logic (LLL) is characterized by means of a non-reflexive accessibility relation. Henceforth, I will call these logics non-reflexive iAML.

Example. Consider the iAML-proof below, based on the premise set $\Gamma=\{\square(q \wedge \sim q)\}$. This proof shows that it is possible to derive from $\Gamma$ the formula $\square \perp$ (with $\perp$ meaning triviality) on the condition $\{\diamond(q \wedge \sim q)\}$. 


$\begin{array}{lllll}1 & \square(q \wedge \sim q) & \text { PREM } & \emptyset & \emptyset \\ 2 & \square \perp & 1 ; \mathrm{RC}_{\text {syn }} / \mathrm{RC}_{\text {sem }} & \{\diamond(q \wedge \sim q)\} & \{q \wedge \sim q\}\end{array}$

As $\square \perp$ is a trivial consequence, line 2 should get marked at some stage of the proof. And indeed, when the accessibility relation of the LLL is reflexive, the proof can always be extended in such a way that line 2 gets marked.

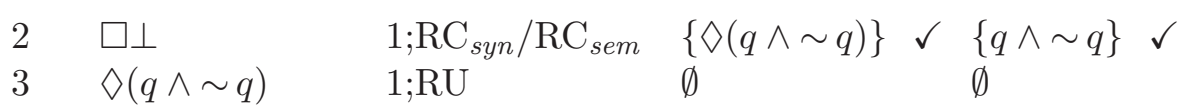

However, when the accessibility relation of the LLL isn't reflexive, the proof cannot be extended in the way shown above (as $\diamond(q \wedge \sim q)$ is not derivable from $\square(q \wedge \sim q))$. Hence, line 2 will remain unmarked, so that the formula on line 2 is considered as finally derivable from the premise set $\Gamma$.

The example clearly shows that when non-reflexive iAML are applied to premise sets such as $\Gamma$, they allow the derivation of all consequences of the form $\square A$. Nevertheless, they do not yield the trivial consequence set. Hence, I have called this feature semi-explosion.

In order to grasp what actually happens in the example (and in equivalent cases), consider things semantically. First, remember that the iAML-models of a premise set are those LLL-models of a premise set that verify the fewest abnormalities. Hence, when the LLL-models verify some necessary inconsistencies (in the example: $\square(q \wedge \sim q)$ ), the LLL-models that verify the fewest abnormalities are the LLL-models for which the set of accessible worlds is empty. ${ }^{6}$ Because of the semantic characterization of necessity, this results in semi-explosion. Moreover, the reason why semi-explosion doesn't occur for reflexive iAML, is also obvious: the set of accessible worlds for reflexive LLL-models is always a non-empty set.

For obvious reasons, the iAML that validate semi-explosion, suffer from the same problems as explosive modal logics (see Section 1).

\footnotetext{
${ }^{6}$ Obviously, I presuppose that the models do not verify any possibilities, in which case the problem of semi-explosion evaporates.
} 
Luckily, it is possible to make some changes to the iAML-framework in order to avoid semi-explosion. But, as this requires some extra work, its characterization is postponed to a follow-up paper.

\section{Application: Paraconsistent Intuitionistic Logic}

To concretize the inconsistency-adaptive modal framework characterized in the previous sections, I will now present a useful application. Specifically, I will show how the framework can be applied to characterize a meaningful paraconsistent version of intuitionistic logic (Int).

Intuitionistic Logic. In [13, pp. 210-211], Epstein showed that the logic Int can be interpreted in the (explosive) modal logic S4. First, consider the following mapping from the intuitionistic language $\mathcal{L}$ (the standard propositional language) to the modal language $\mathcal{L}^{\mathcal{M}}$ :

$$
\begin{aligned}
A^{*} & =\square A \quad(\text { for } A \in \mathcal{S}) \\
(A \wedge B)^{*} & =A^{*} \wedge B^{*} \\
(A \vee B)^{*} & =A^{*} \vee B^{*} \\
(A \supset B)^{*} & =\square\left(A^{*} \supset B^{*}\right) \\
(\sim A)^{*} & =\square \sim\left(A^{*}\right)
\end{aligned}
$$

and

Definition 28. $\Gamma^{*}=\left\{A^{*} \mid A \in \Gamma\right\}$.

Next, consider Theorem 9 below. It spells out how to interpret the logic Int in the logic $\mathbf{S} 4$.

Theorem $9 . \Gamma \vdash_{\text {Int }} A$ iff $\Gamma^{*} \vdash_{\mathbf{S} 4} A^{*}$.

Paraconsistent Intuitionistic Logic. In order to characterize a meaningful paraconsistent version of intuitionistic logic (let's call it pInt), the logic $\mathbf{S} 4$ in Theorem 9 can be replaced by the inconsistency-adaptive modal logic $\mathbf{i A S} \mathbf{4} \overline{\mathbf{u}} \mathbf{N} \mathbf{s}_{\text {syn }}^{\mathbf{r}}$ that is characterized by the lower limit logic $\mathbf{S} 4 \overline{\mathbf{u}} \mathbf{N s},{ }^{7}$ the set of abnormalities $\Omega_{\text {syn }}$ and the reliability strategy. ${ }^{8}$

\footnotetext{
${ }^{7}$ The logic $\mathbf{S} 4 \overline{\mathbf{u}} \mathbf{N s}$ is completely equivalent to the logic $\mathbf{T} \overline{\mathbf{u}} \mathbf{N s}$ with the exception of its accessibility relation. While the accessibility relation of $\mathbf{T} \overline{\mathbf{u}} \mathbf{N s}$ is merely reflexive, the accessibility relation of $\mathbf{S} \mathbf{4} \overline{\mathbf{u}} \mathbf{N s}$ is both reflexive and transitive.

${ }^{8} \mathrm{I}$ have opted for the syntactic approach, but the semantic approach will obviously do as well.
} 
TheOREM 10. $\Gamma \vdash_{\text {pInt }} A$ iff $\Gamma^{*} \vdash_{\mathbf{i A S} 4 \overline{\mathbf{u}} \mathbf{s s}_{s y n}^{\mathrm{r}}} A^{*}$.

The main advantage of the logic pInt consists in the fact that for consistent premise sets, it yields the same consequences as the logic Int, while for inconsistent premise sets, it only yields those consequences that can be derived by interpreting the premise set as consistently as possible. As this is most clearly illustrated by means of an example, let's have a look at the one below.

Example. Consider the premise set $\Gamma=\{p \supset q, p \supset \sim q, p, r \supset s$, $r\}$, which is mapped to the premise set $\Gamma^{*}=\{\square(\square p \supset \square q), \square(\square p \supset$ $\square(\sim(\square q))), \square p, \square(\square r \supset \square s), \square r\}$. The $\mathbf{i A S} 4 \overline{\mathbf{u}} \mathbf{N} \mathbf{s}_{s y n}^{\mathbf{r}}$-proof now proceeds as follows.

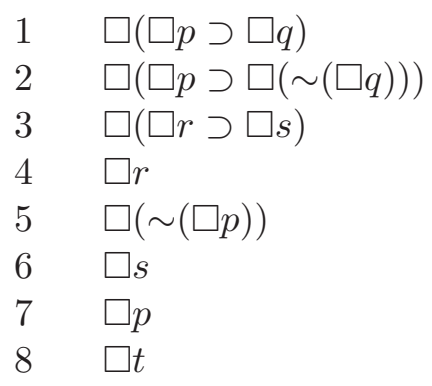

$$
\begin{array}{ll}
\text {-;PREM } & \emptyset \\
- \text {-PREM } & \emptyset \\
- \text {-PREM } & \emptyset \\
\text {-;PREM } & \emptyset \\
1,2 ; \mathrm{RC}_{\text {syn }} & \{\oslash(q \wedge \sim q)\} \\
3,4 ; \mathrm{RC}_{\text {syn }} & \{\diamond(r \wedge \sim r)\} \\
-; \mathrm{PREM}^{\mathrm{PR}} & \emptyset \\
5,7 ; \mathrm{RC}_{\text {syn }} & \{\diamond(q \wedge \sim q), \diamond(p \wedge \sim p)\}
\end{array}
$$

At stage 8 of the proof, all lines are unmarked. However, line 5 and line 8 get marked when the proof is extended in the following way.

$$
\begin{array}{lllll}
5 & \square(\sim(\square p)) & 1,2 ; \mathrm{RC}_{\text {syn }} & \{\diamond(q \wedge \sim q)\} & \checkmark \\
\ldots & \ldots & \ldots & \ldots \\
8 & \square t & 5,7 ; \mathrm{RC}_{\text {syn }} & \{\diamond(q \wedge \sim q), \diamond(p \wedge \sim p)\} & \checkmark \\
9 & \diamond(p \wedge \sim p) \vee \diamond(q \wedge \sim q) & 1,2,7 ; \mathrm{RU} & \emptyset
\end{array}
$$

Moreover, as lines 5 and 8 cannot become unmarked again, the formulas on those lines are not finally derivable from the premise set $\Gamma^{*}$. On the other hand, the conditionally derived formula on line 6 is finally derivable from $\Gamma^{*}$, as the proof cannot be extended in such a way that line 6 will be marked.

\section{Conclusion}

In this paper, I have characterized a new class of inconsistency-adaptive logics, namely inconsistency-adaptive modal logics. In general, these 
combine the paraconsistency of their lower limit logic (a paraconsistent modal logic) with the deductive strength of their upper limit logic (an explosive modal logic). Hence, in case of inconsistent premise sets, they avoid explosion, but still allow the derivation of sufficient consequences to explicate human reasoning in a realistic way. ${ }^{9}$

\section{References}

[1] Diderik Batens. "Inconsistency-adaptive logics", pp. 445-472 in: E. Orłowska, editor, Logic at Work. Essays Dedicated to the Memory of Helena Rasiowa. Physica Verlag (Springer), Heidelberg, New York, 1999.

[2] Diderik Batens, "A survey of inconsistency-adaptive logics", pp. 49-73 in: D. Batens, C. Mortensen, G. Priest, and J.P. Van Bendegem, editors, Frontiers of Paraconsistent Logic. Research Studies Press, Baldock, UK, 2000 .

[3] Diderik Batens. "A general characterization of adaptive logics", Logique et Analyse 173-175 (2001): 45-68.

[4] Diderik Batens, "A strengthening of the Rescher-Manor consequence relations", Logique et Analyse 183-184 (2003): 289-313.

[5] Diderik Batens, "A universal logic approach to adaptive logics", Logica Universalis 1 (2007): 221-242.

[6] Diderik Batens. "Aspects of the dynamics of discussions and logics handling them". To appear.

[7] Diderik Batens and Kristof De Clercq, "A rich paraconsistent extension of full positive logic", Logique et Analyse 185-188 (2004): 227-257.

[8] Diderik Batens and Lieven Haesaert, "On classical adaptive logics of induction", Logique et Analyse, 173-175 (2001): 255-290.

[9] Diderik Batens and Joke Meheus, "Recent results by the inconsistencyadaptive labourers", pp. 274-290 in: J.-Y. Béziau, W. Carnielli, and D. Gabbay, editors, Handbook of Paraconsistency. College Publications, London, 2007.

[10] Diderik Batens, Joke Meheus, and Dagmar Provijn. "An adaptive characterization of signed systems for paraconsistent reasoning". To appear.

\footnotetext{
${ }^{9}$ Most unpublished papers in the reference section are available on the following internet address: http://logica.ugent.be/writings/.
} 
[11] Diderik Batens, Joke Meheus, Dagmar Provijn, and Liza Verhoeven, "Some adaptive logics for diagnosis", Logic and Logical Philosophy 11-12 (2003): 39-65.

[12] Marcelo Coniglio, "Logics for deontic inconsistency", CLE e-prints, 7(4), 2007.

[13] Richard Epstein. Semantic Foundations of Logic, vol. 1. Kluwer, Dordrecht, 1990.

[14] Lou Goble, "Paraconsistent modal logic", Logique et Analyse 193 (2006): $3-29$.

[15] Hans Lycke, "Fitch-style natural deduction for modal paralogics", Logique et Analyse 207 (2009): 193-218.

[16] Hans Lycke, Relevance in Reasoning. The Adaptive Logics Approach, PhD thesis, Ghent University, Gent, Belgium, 2007.

[17] Joke Meheus, "An adaptive logic for pragmatic truth", pp. 167-185 in: W. Carnielli, M. Coniglio, and I. M. Loffredo D'Ottaviano, editors, Paraconsistency. The Logical Way to the Inconsistent. Marcel Dekker, New York, 2002.

[18] Joke Meheus, "Paraconsistent compatibility", Logique et Analyse 183-184 (2003): 251-287.

[19] Joke Meheus, "An adaptive logic based on Jaśkowski's approach to paraconsistency", Journal of Philosophical Logic 35 (2006): 539-567.

[20] Graham Priest, "Logic of paradox", Journal of Philosophical Logic 8 (1979): 219-241.

[21] Graham Priest, An Introduction to Non-Classical Logic. Cambridge University Press, Cambridge, 2001.

[22] Graham Priest, "Paraconsistent logic", pp. 287-393 in: D. Gabbay and F. Guenthner, editors, Handbook of Philosophical Logic, vol. 6. Kluwer, Dordrecht/Boston/London, 2nd edition, 2002.

[23] Giuseppe Primiero and Joke Meheus, "Majority merging by adaptive counting", Synthese (KRA-series), 165 (2008): 203-223.

HANS LYCKE

Centre for Logic and Philosophy of Science

Ghent University

Ghent, Belgium

Hans. Lycke@Ugent . be 